\title{
The Historical Archaeology of the Chinese in Far North Queensland
}

\author{
Heather Burke and Gordon Grimwade \\ Department of Archaeology, Flinders University, GPO Box 2100, Adelaide SA 5001, Australia \\ heather.burke@flinders.edu.au,ggrimwade@bigpond.com
}

\begin{abstract}
The first Overseas Chinese immigrants to Far North Queensland (FNQ) arrived in 1868 and within 20 years had established a wide range of settlements keyed directly into Chinese merchant supply networks. Historical archaeology on some of these sites has been carried out since the mid-1980s, but has largely been consultancy-driven, creating data that are patchy, skewed towards urban centres and often non-comparable. Such comparisons as can be drawn relating to dining and drinking behaviours, however, show assemblages dominated by traditional ceramic bowls and a high proportion of plain celadon and Four Seasons decorated wares, but an early and decided preference for European alcohols. Continued adherence to Chinese cultural preferences relating to food but not to alcohol suggests that concepts of identity and the construction of the self may have been constructed differently in each arena. While limited in depth, the archaeology of the Overseas Chinese in FNQ highlights critical gaps and provides a preliminary platform from which to identify future research directions, particularly a need to supplement impact assessment-related studies with detailed surface recording and/or carefully targeted open area excavations in order to advance knowledge beyond basic presence/absence questions.
\end{abstract}

\section{Introduction}

The Chinese migrations of the nineteenth century constituted one of the world's largest population movements (Voss and Allen 2008:6). The majority travelled from areas of southeastern China (Guangdong) as bonded labour, according to a credit ticket system that mortgaged the cost of their passage against family or clan land and bound them to their creditor until either their passage was paid with interest or a specified period had elapsed (Bowen 2011:31; Reeves 2010:184). The communities at the heart of this system were known as qiaoxiang (literally 'sojourner's village' or 'emigrant community') and were part of a complex reciprocal network that specialised in sending men abroad and then relying on them to send regular remittances back to China (Pan 1998:27). Whether such emigrants were pushed or pulled to leave, the Overseas Chinese (i.e. Chinese living abroad) were predominantly men in search of opportunity. The second half of the nineteenth century saw more than 2.5 million such departures from China, particularly for the gold rush societies that formed around the Pacific Rim from the 1850s onwards.

This paper provides an overview of the historical archaeology of the Overseas Chinese who lived in Far North Queensland (FNQ) between 1868 and the 1930s. The study area stretches from Cardwell in the south to Cooktown in the north, and west to Normanton (Figure 1). Building on a brief history of the Chinese in FNQ, this paper presents a review of previous historical archaeological work and then focuses on comparisons of the most durable data - the core categories of glass and ceramics - since the behaviours around food and drink generated much of the material evidence present at sites and these artefact classes have been the most commonly recorded and are therefore the most comparable between projects.

\section{The Chinese in Far North Queensland}

The Chinese who came to FNQ were following a wellestablished - and much wider - pattern of seeking material advantage and individual autonomy (Reeves
2010:189). The first to arrive were following a trail of mineral discoveries that began at the Cape River (south of Charters Towers) in 1867, and moved swiftly to the Gilbert River (200km northwest of Charters Towers) in the following year. By 1871 there was a thriving population of 430 Chinese in Gilberton and census data clearly show the ebb and flow of population as people followed various labour opportunities to Etheridge (later renamed Georgetown) in 1872, the Palmer River and Cooktown in 1873, the Hodgkinson, Atherton, Port Douglas and Cairns in 1876/1877, and Herberton, Croydon, the Johnstone River and Geraldton (later renamed Innisfail) in the 1880s.

The Palmer River gold rush was the most significant event in this history. The first Overseas Chinese to arrive here probably came overland from Gilberton in late 1873/early 1874 as a consequence of local Aboriginalsettler violence in Gilberton (The Brisbane Courier 6 January 1874) and the lure attached to the opening of the Palmer field. In July 1874 more had arrived via steamer from Rockhampton and by January 1875 the first passengers had arrived directly from Hong Kong to the new port of Cooktown (Kirkman 1993). The ensuing monthly international steamer service swelled the population until Chinese on the Palmer outnumbered Europeans three to one (Comber 1991:10). In 1876, 77\% of all Overseas Chinese in Queensland lived in the far north (Table 1) and between 1877 and 1880 the Chinese miners on the Palmer constituted the largest Chinese community in the Colony. Bell (1997:6) has estimated that in 1877 there were probably more Chinese on the Palmer than Europeans in the entire northern half of Australia, a suggestion endorsed by Jones (1997:112).

This mass migration affected FNQ for decades, both demographically and economically. Fitzgerald (2007:55) cites figures for 1883 which indicate that half or more of the populations of the Cairns, Port Douglas, Innisfail and Atherton regions were of Chinese descent. Most of the men who had arrived in the mid-1870s were young and by the 1881 census the dominant demographic group consisted of men aged 20-35 (Queensland Registrar 


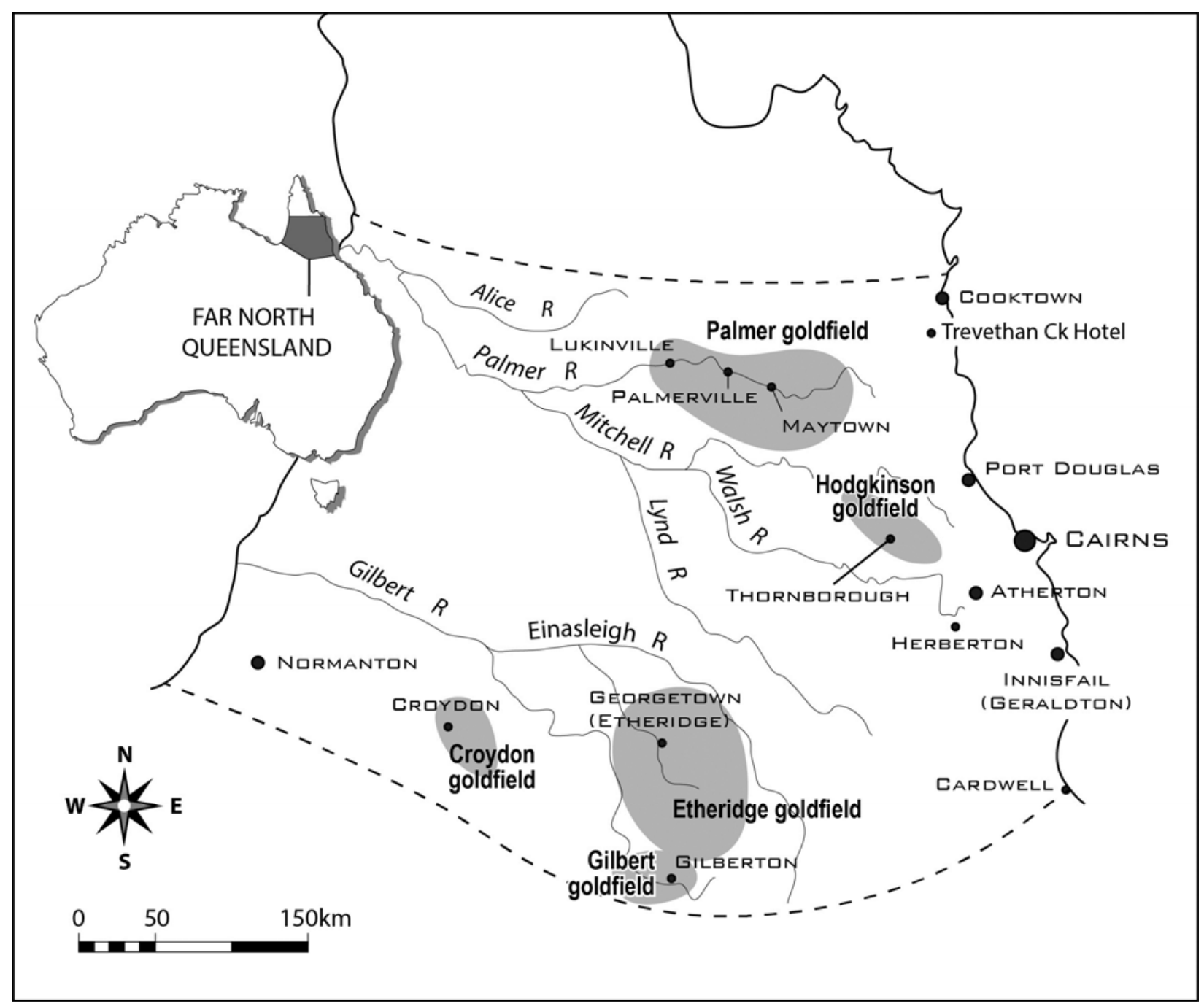

Figure 1. Far North Queensland, including sites and locations mentioned in the text.

General's Office 1881:19). Relatively few Chinese women were part of Overseas communities, although there were always more in the stable settlements of Cooktown, Cairns and Atherton (Table 1).

\section{Key Facets of the Chinese Experience}

Much of the Overseas Chinese experience was constructed within a prejudicial and sometimes antagonistic matrix, such that processes of self-definition and collective identification would have been continually negotiated in response to changing social and political circumstances. Within Chinese communities identification was similarly complex, relating to the effects of both broader structuring principles of identity, class, race and gender within Chinese and European (i.e. capitalist) societies, the status and class connotations of district of origin, relationships between kin, hierarchical distinctions between the merchant elite and others, and political divisions that affected internal and external outlooks. A key facet of the Chinese experience was the dynamic generated by a life predicated around constant mobility, but with a stable core that nevertheless sought to build community in a number of ways.

\section{Mobility}

Fitzgerald (2007:54) has argued that mobility was the most exceptional feature of Chinese-Australian settlement. Locally and regionally, changes in labour opportunities saw many Overseas Chinese shift their place of residence and source of employment seasonally. This meant that many small businesses changed hands regularly; in Cairns, for example, this could happen as rapidly as every three to four years (May 1984:Appendix H). According to Rains (2005:84), Chinese businesses were intended to be short-lived, as well as relatively small-scale and self-sufficient, since they were focussed on amassing wealth for the betterment of family members. Mobility of domicile was also common, with May (1984:101) suggesting that individuals might move to different lodgings within the same town every few weeks, often for no express reason. Such levels of mobility were common amongst Europeans immigrants as well, however. Figures for the European town of Marulan, New South Wales, for example, indicate mobility rates of up to $90 \%$ throughout the mid-to-late nineteenth century, and an almost total turnover of population every 10 years (Gojak 2009), suggesting that mobility rates generally may be underappreciated in historical archaeological analysis.

At least some of the strength of Chinese identity was linked to the ultimate goal of returning to China and census figures suggest that many had chosen to leave Australia by the age of 50 (May 2001:211). Many also made round trips, travelling between Pacific Rim 
Table 1. Numbers of Chinese men and women (adult and minors) in Far North Queensland, 1861-1901 (Historical Census and Colonial Data Archive 2013; Government Statistician 2013).

\begin{tabular}{|c|c|c|c|c|c|c|c|c|c|}
\hline \multirow[b]{2}{*}{ Region } & \multicolumn{9}{|c|}{ Year } \\
\hline & Sex & 1861 & 1868 & 1871 & 1876 & 1881 & 1886 & 1891 & 1901 \\
\hline \multirow[t]{2}{*}{ Burke Census District } & Male & 0 & $\begin{array}{r}51 \\
\text { [14 Burketown] }\end{array}$ & 39 & 16 & 97 & $\begin{array}{r}174 \\
\text { [15 Croydon] }\end{array}$ & 341 & 52 \\
\hline & Female & 0 & 0 & 0 & 0 & 0 & 0 & 3 & 1 \\
\hline \multirow[t]{2}{*}{$\begin{array}{l}\text { Gilbert/Etheridge Census } \\
\text { District }\end{array}$} & Male & 0 & 0 & 430 & 48 & 362 & $\begin{array}{r}202 \\
\text { [56 Gilberton; } \\
43 \text { Georgetown] }\end{array}$ & 197 & 176 \\
\hline & Female & 0 & 0 & 0 & 0 & 0 & 1 & 0 & 9 \\
\hline \multirow{2}{*}{ Cook Census District } & Male & 0 & 1 & 1 & 1350 & 1249 & 1357 & 579 & 400 \\
\hline & Female & 0 & 0 & 0 & 0 & 10 & 17 & 6 & 12 \\
\hline \multirow[t]{2}{*}{ Palmer Census District } & Male & 0 & 0 & 0 & 6671 & 5133 & $\begin{array}{r}967 \\
\text { [134 Maytown; } \\
99 \text { Sandy Ck; } \\
14 \text { Palmerville; } \\
18 \text { Granite Ck; } \\
19 \text { Palmer River; } \\
7 \text { Coen River] }\end{array}$ & 554 & 384 \\
\hline & Female & 0 & 0 & 0 & 0 & 3 & 4 & 1 & 0 \\
\hline \multirow{2}{*}{$\begin{array}{l}\text { Woothakata Census } \\
\text { District }\end{array}$} & Male & 0 & 0 & 0 & 0 & 0 & 122 & 206 & 92 \\
\hline & Female & 0 & 0 & 0 & 0 & 0 & 0 & 0 & 0 \\
\hline \multirow{2}{*}{$\begin{array}{l}\text { Cardwell Census } \\
\text { District }\end{array}$} & Male & 0 & 2 & 0 & 1 & 27 & 331 & 227 & 310 \\
\hline & Female & 0 & 0 & 0 & 0 & 0 & 0 & 1 & 1 \\
\hline \multirow{2}{*}{ Cairns Census District } & Male & 0 & 0 & 0 & 0 & 0 & 1397 & 1593 & 2017 \\
\hline & Female & 0 & 0 & 0 & 0 & 0 & 0 & 0 & 61 \\
\hline \multirow{2}{*}{$\begin{array}{l}\text { Herberton Census } \\
\text { District }\end{array}$} & Male & 0 & 0 & 0 & 0 & 0 & 259 & 344 & 483 \\
\hline & Female & 0 & 0 & 0 & 0 & 0 & 1 & 0 & 1 \\
\hline FNQ Subtotal & & 0 & 54 & 470 & 8086 & 6881 & 4832 & 4052 & 3999 \\
\hline \multirow{3}{*}{ Queensland } & Male & 537 & 2621 & 3304 & 10399 & 11206 & 10444 & 8497 & 8137 \\
\hline & Female & 1 & 8 & 1 & 13 & 23 & 56 & 47 & 782 \\
\hline & Total & 538 & 2629 & 3305 & 10412 & 11229 & 10500 & 8544 & 8919 \\
\hline \multirow{3}{*}{ Australia } & Male & 38247 & - & 28302 & - & 38274 & - & 35523 & 29153 \\
\hline & Female & 11 & - & 44 & - & 259 & - & 298 & 474 \\
\hline & Total & 38258 & - & 28346 & - & 38533 & - & 35821 & 29627 \\
\hline $\begin{array}{l}\text { FNQ as a \% of } \\
\text { Queensland }\end{array}$ & & 0 & 2.05 & 14.22 & 77.66 & 61.28 & 46.02 & 47.43 & 44.84 \\
\hline FNQ as a \% of Australia & & 0 & 0 & 1.66 & 0 & 17.86 & 0 & 11.31 & 13.50 \\
\hline
\end{tabular}

countries or to and from China over the course of their lives (Reeves 2010; see also Williams 1999:16). Very few (approximately 4\%) succeeded in becoming naturalised Australian citizens (Wong Hoy 2004:161), although others would have had children recognised as Australian by birth. Many more who developed relationships with Aboriginal women would have had children who were not recognised as 'Australian' in any legal sense at the time. This complexity has led Chan (1999:2-3) to posit three levels of Overseas Chinese identity: the Huaqiao - those who returned; the Huashang - merchants who succeeded abroad through business; and the Huayi - Chinese who took out foreign citizenship or were born overseas. As a result, both Chan (1999) and Curthoys (2001:121) have argued that 'Chineseness' could equally be structured in terms of a real or mythologised China, an association with the host nation or adopted country, or an alliance with the Diaspora itself.

\section{Community and Identity}

Throughout the nineteenth century there were two ways in which 'community' was built amongst the Overseas Chinese: psychologically through a complex web of social support networks that linked Overseas Chinese to their home villages, as well as to each other, and literally through the construction of physical 'hub' settlements Burke \& Grimwade (often referred to by Europeans as 'Chinatowns') that provided a range of sacred and secular services, including temples, stores and accommodation.

One of the most important ways in which psychological bonds within Overseas Chinese communities were constructed was through the activities of benevolent organisations based on a migrant's village, county or district of origin. People belonged to such 'native place' associations by virtue of birth, and were able to access practical, spiritual and social assistance via their networks. In Guangdong the Toi Shan, Sze Yap and Sam Yap associations were the three largest such organisations (Reeves 2010:182; Reeves and Mountford 2011:124) and their Australian counterparts were first consolidated on the Victorian goldfields in the 1850s. As elsewhere, the majority of the Overseas Chinese in FNQ came from three areas of Guangdong: most from the Chung Shan (Zhongshan) district, some from the three districts of Sze Yap and some from the three districts of Sam Yap (May 1984:64, 1993a:262-263). Identity constructed according to birthplace often led to sharp divisions (Reeves and Mountford 2011:118). In 1878 at Lukinville on the Palmer, for example, hostilities broke out between rival factions from Guangzhou (Canton Sam Yap) and Macao (Chung Shan) (Kirkman 1984:231232). Similarly, in Cairns the Chung Shan and Sze Yap q a $r$ | Vol. 16 | 2013 | 123 


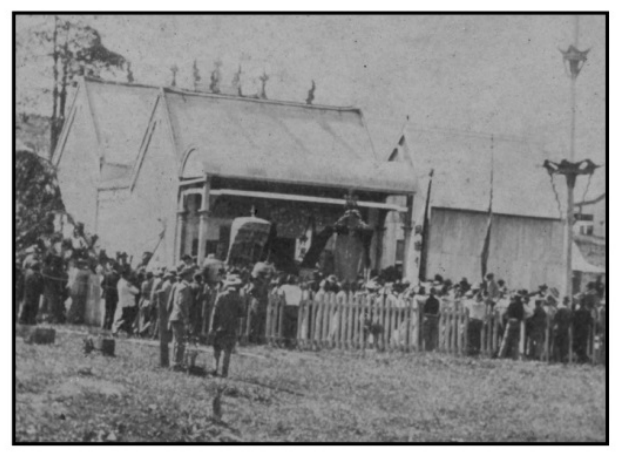

a. First Innisfail temple, built late 1800 s (Photo: Courtesy National Library of Australia vn3551238)

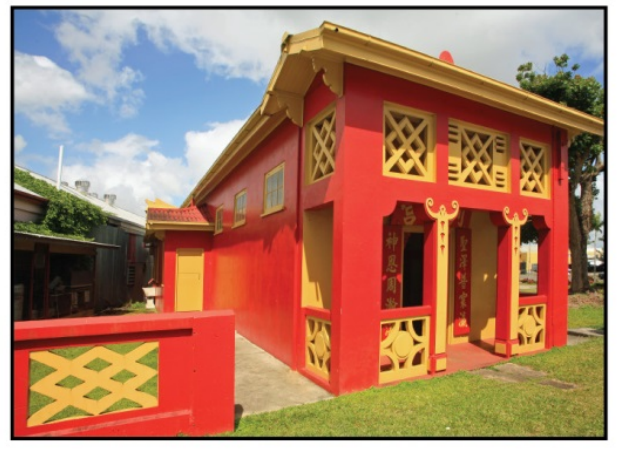

c. Second Innisfail temple, built c1940

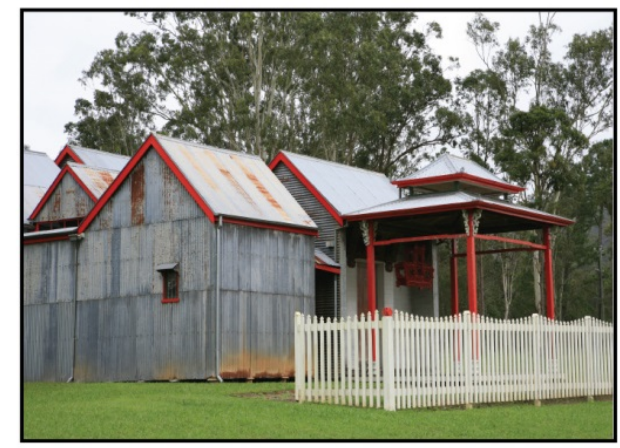

b. Atherton temple, built 1903

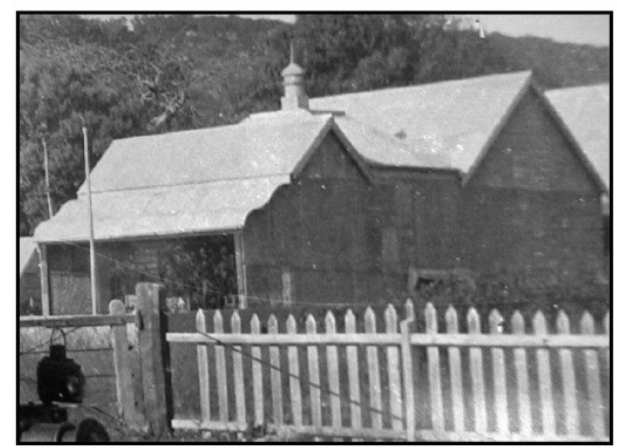

d. Cooktown temple. This is probably the second temple, built c1880

Figure 2. FNQ temples followed an architectural form once common across rural Australasia and North America.

maintained separate temples and stores, a distinction which arose partly because the dialect spoken by Chung Shan immigrants was 'barely intelligible' to the other groups (May 1984:64). As a result, immigrants from different districts could have largely separate social and economic arrangements (May 1993a:267, 2001:211). Fitzgerald (2005:97), however, has argued for a rapid transformation of such organisations in the colonial context, such that, as early as the 1870 s they had begun to move away from direct native-place or clan ties and relate more specifically to their place of domicile in Australia, thus becoming more pan-Chinese.

Many of the key services offered by native place associations were accessible within hub settlements - the Chinatowns that formed in many parts of Australia from the 1860s onwards. Gilberton was the first in FNQ, established by early 1870 (The Queenslander 14 May 1870; The Brisbane Courier 28 May 1870), followed quickly by Cooktown, Georgetown and the Palmer by 1875, Atherton by 1877, Cairns and Port Douglas by 1880 and Croydon in 1885. Hum Lee (1960:58) has argued that 390 people was the critical mass for a viable Chinatown; below this, essential services, such as temples, could not be sustained. Using temples as a measure there were at least nine such viable hubs in FNQ throughout the nineteenth century: Cooktown (where two or three temples were built in the late nineteenth century), the Palmer (one temple, c.1878), Etheridge/ Georgetown (which had at least two temples, one constructed in 1891 and another in 1905), Port Douglas (c.1880s), Cairns (two separate temples, one erected in 1886 and the other c.1898), Croydon (one temple built in 1897 and its replacement in 1903), Thornborough (1898), Innisfail (one temple in the late 1800s and its replacement c.1940) and Atherton (1903) (see Figure 8). Only Hou Wang Miau in Atherton (1903), and Lit Sing Gung, the second temple built in Innisfail (c.1940), survive today. The only major artefact collections derive from the Atherton, Cairns, Innisfail and, possibly, Georgetown temples, although the site of the Croydon temple has been extensively recorded (Grimwade 2003).

Photographic evidence suggests that FNQ temples were broadly similar to the national trend in form and layout - long, narrow buildings with a community hall attached to one side - conforming to the three house temple design, which provided separate community and worship space (Figure 2). A comparative study of the Atherton and Croydon temples (Grimwade 2003) noted marked similarities between the two buildings, although the Atherton temple's distinctive front pagoda section was a relatively uncommon feature. The second Innisfail temple, by contrast, was both later in date and distinct from its earlier counterparts. It not only incorporates masonry brick, ceramic tiled floors and concrete rendering, but has an unorthodox layout, with a single area for worship and a community room/residence at the 
rear of the temple, rather than to one side. It possesses neither a 'heavenly well' nor a lantern roof. The building blends art deco characteristics with traditional Chinese form and elements but demonstrates distinct cross-cultural influences and ignores some fundamentals of standard temple design (Figure 2).

Pig ovens, designed to roast whole pigs on feast days, were an integral feature of many temple sites and were located adjacent to the temples in Croydon, Atherton, Cairns, Etheridge, Innisfail, Port Douglas and Thornborough. Although research suggests that Chinese cooking ovens were more widely represented in Pacific Rim countries than previously thought (Mainery 2001), particularly by the twentieth century (Chan 2007; Grimwade 2008), both Bell (1995) and Chan (2007) have pointed out the uniqueness of ovens in the nineteenth century trans-Tasman context. Chan (2007:166) has speculated that their presence may have served as an indicator of 'substantial and mature' communities, while noting that their precise origins remain unknown.

\section{Previous Archaeological Work on the Overseas Chinese in FNQ}

While the Overseas Chinese were spread widely across the FNQ landscape, much of the archaeological work has been consultancy-driven, responding to development needs in major centres, or the exploitation of mineral deposits in the Palmer. This has created certain problems, notably data that are skewed towards the urban centres, particularly the residential areas of Cairns and Atherton, and a tendency to focus on site locations with only summary descriptions of contents. There have only been six dedicated research projects across the region: a graduate diploma thesis on Atherton's Hou Wang Temple (Ibrahim 1981), some early work on the Palmer (Jack et al. 1984), a graduate diploma thesis (McCrae 2001), Honours thesis (Rains 1999) and PhD (Rains 2005) on Cooktown, and an Honours thesis on Atherton Chinatown (Dunk 2010). As a result, the bulk of the work has not been designed to consider behaviourally-oriented research questions (but note that some academic papers have been derived from consultancy and other work, e.g. Comber 1995; Grimwade 2003, 2007, 2008). Only three sites have been excavated: Ah Toy's garden on the Palmer River (Jack et al. 1984), Atherton Chinatown (Cutler and Reynolds 1991; Rowney 2003; Scott and Cutler 1993) and Cairns Chinatown (Grimwade and Rowney 2004), although surface collections have also been carried out at Atherton and Croydon Chinatowns. In terms of contents data, catalogues only exist for the more recently excavated assemblages from Atherton Chinatown (Dunk 2010; Rowney 2003), Cairns Chinatown (Grimwade and Rowney 2004) and Cooktown (Grimwade et al. 2007).

\section{Palmer River (1874-c.1920s)}

While the Palmer hosted the region's most significant early concentrations of Overseas Chinese, in comparison to the number of people and the density of settlement, negligible archaeological work has been conducted (Figure 3). The earliest was the 1984 excavation of Ah Toy's garden (Jack et al. 1984). Occupied between 1883 and 1934, excavations and surface collection recovered numerous agricultural implements, some domestic ceramics and glass, and recorded the remains of a hut, two bottle dumps, a dam and a water race. Unfortunately, the published work on this site (Jack et al. 1984) contains no quantified data, although it does note that most of the bottles were liquor and beer bottles, including Dutch schnapps, Scotch whisky, English and Dutch gin and Japanese, Philippine and German beer (Jack et al. 1984:56).

In 1986, in response to pressure from the Palmer River Historic Preservation Society, the Queensland Government designated part of the former goldfield an historic reserve (R16) (see Figure 3). Mining was only permitted subject to an archaeological clearance report, although several key areas were omitted from the reserve, including the South Palmer, Byerstown and Groganville. In 1990 the then Department of Environment and Heritage commissioned two studies of R16 to identify its historic heritage places. Grimwade's (1990) desktop study identified 211 sites. Of these, 82 were considered to have Chinese origins, 59 were considered European, five both European and Chinese, and five were post-contact Indigenous sites, while 60 were indeterminate. Subsequent work on the Palmer River Goldfields Heritage Sites Study (Comber 1991) reduced the total to 205, since several, when investigated in the field, were considered part of the same site.

Subsequent reports indicate that Grimwade (1990) and Comber (1991) fell short of identifying 'all' R16 sites. For example, seven of the nine reports prepared between 1993 and 1996 reported 85 historical archaeological sites, 46 of which were in neither Grimwade (1990) nor Comber (1991). At least 25 archaeological assessments were undertaken in R16 between 1987-2007. In reporting on identifiable Chinese sites, most recorded isolated dwelling (hut) sites, graves (Figure 4), alluvial workings, market gardens and larger campsites. Associated artefact scatters included a diversity of ceramics, opium cans, European alcohol bottles and discarded hand tools. Many ovens (Figure 5) have also been recorded in the Palmer, but, as with those at Pine Creek, Northern Territory (Bjornskov 2001), are not associated with known temple sites. Comber (1991:23) noted that several of the Palmer ovens contained 'broken and charred bone material either inside or surrounding [them] ... The majority were located near habitation sites, which indicates that they were more likely to have been used for cooking'. The lack of more comprehensive research on these structures leaves open the question of whether or not all, or some, were domestic ovens as opposed to forges or bakery ovens. A cluster of such structures on Cannibal Creek, south of R16, has been casually noted in the past but remains to be more thoroughly recorded.

Many sites in the Palmer cannot be confidently associated with the Overseas Chinese. Of a total of 235 sites noted in the 25 reports accessed for this study (Table 2), 56 can be confidently considered 'Chinese' (Figure 3 inset), while six were shared sites (townships and cemeteries). Fifty-one can be considered to be primarily European sites on the basis of the style of mining being undertaken, since in the Palmer both hard rock and alluvial mines were the work of Europeans, while the Chinese concentrated on alluvial mining. Two Aboriginal sites were noted in one report, but cultural origins for a total of 120 sites were considered too difficult to determine. 


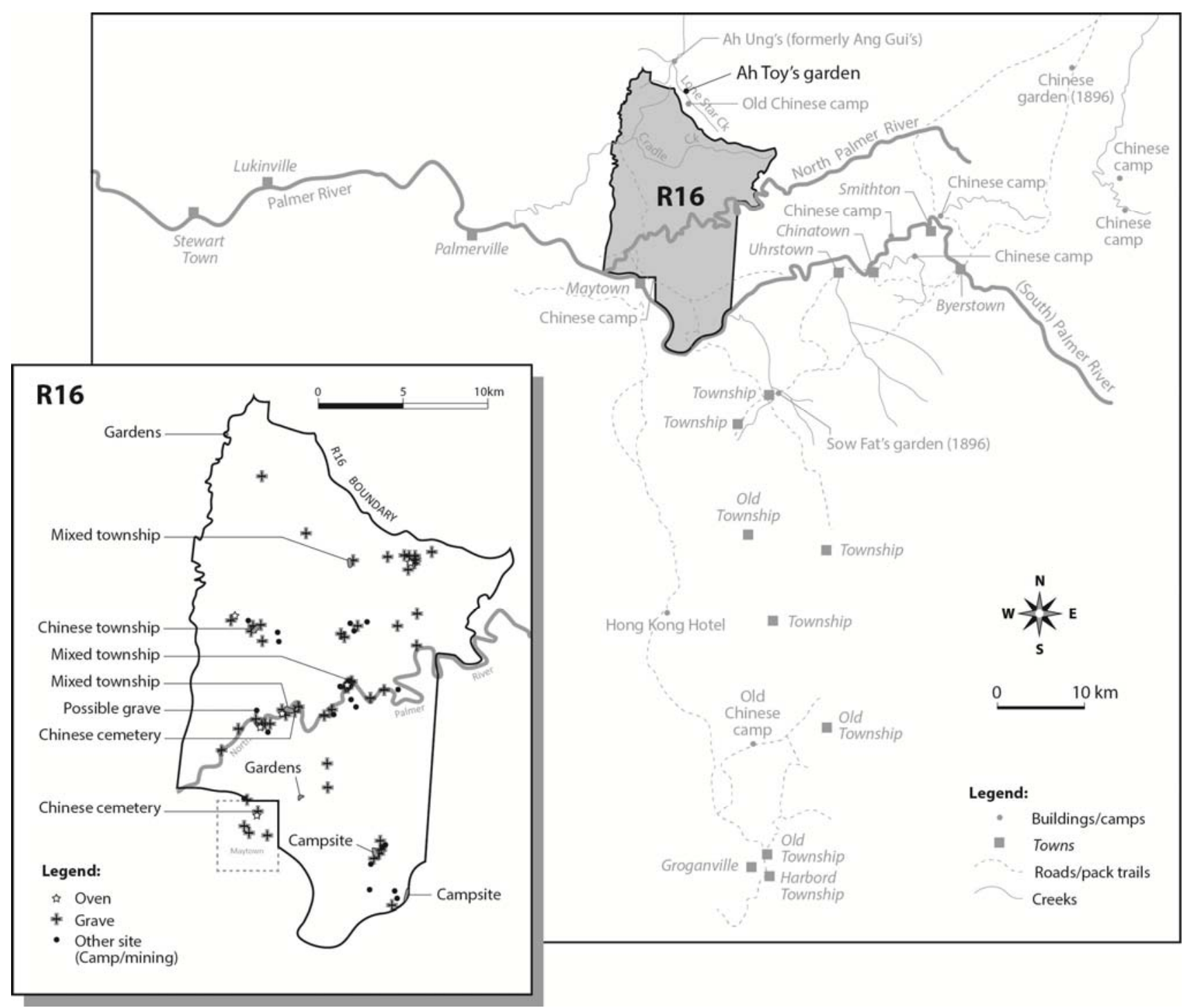

Figure 3. Historical towns and other places on the Palmer River Goldfield. Features, place names and sites in grey are taken from Jack $(1896,1899,1921)$. Inset: Chinese sites recorded in R16.

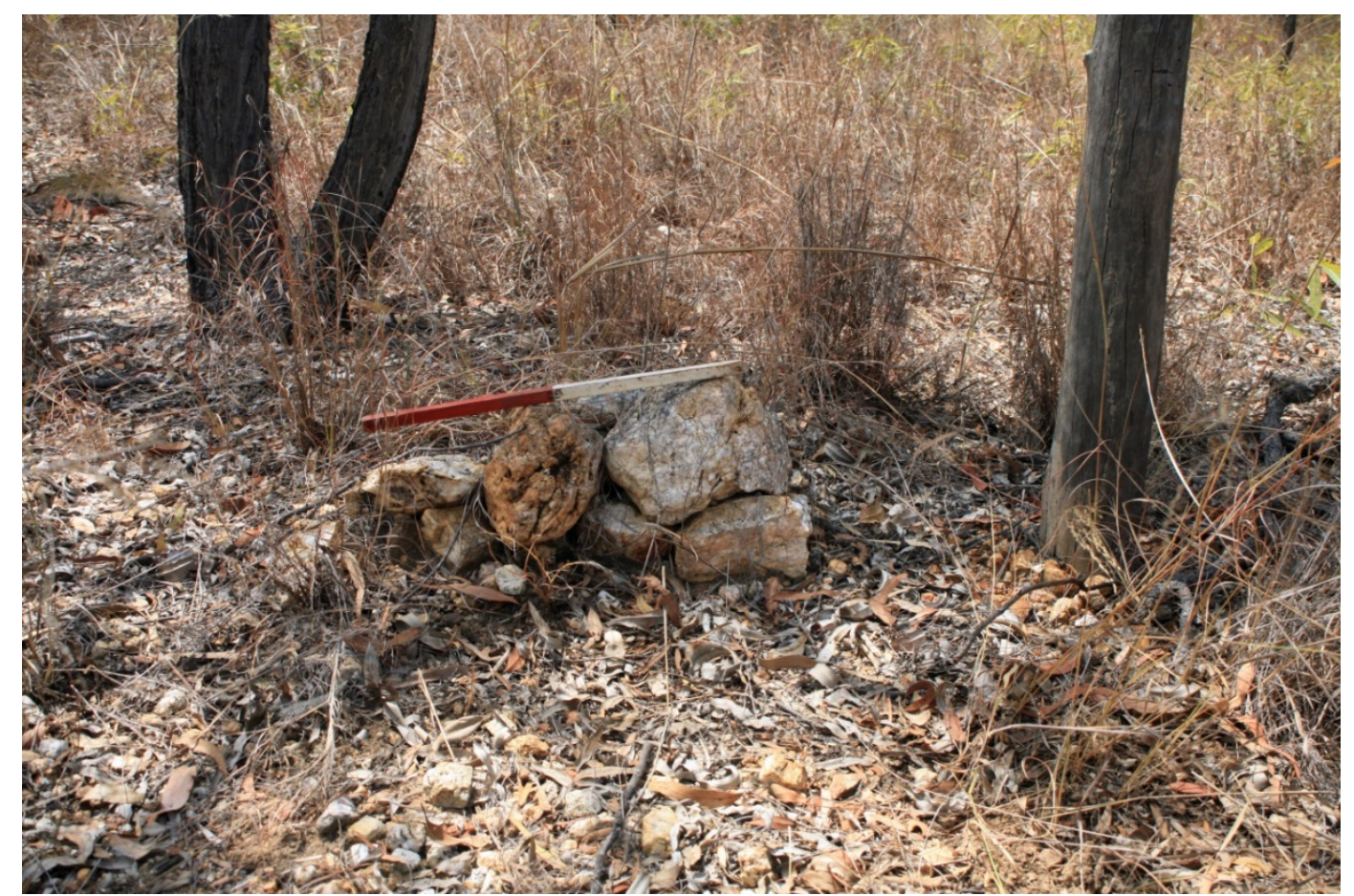

Figure 4. Isolated Chinese burials, identifiable by mounds of footstones, lie along many of the river valleys of the Palmer River Goldfield (Photograph: Gordon Grimwade). 


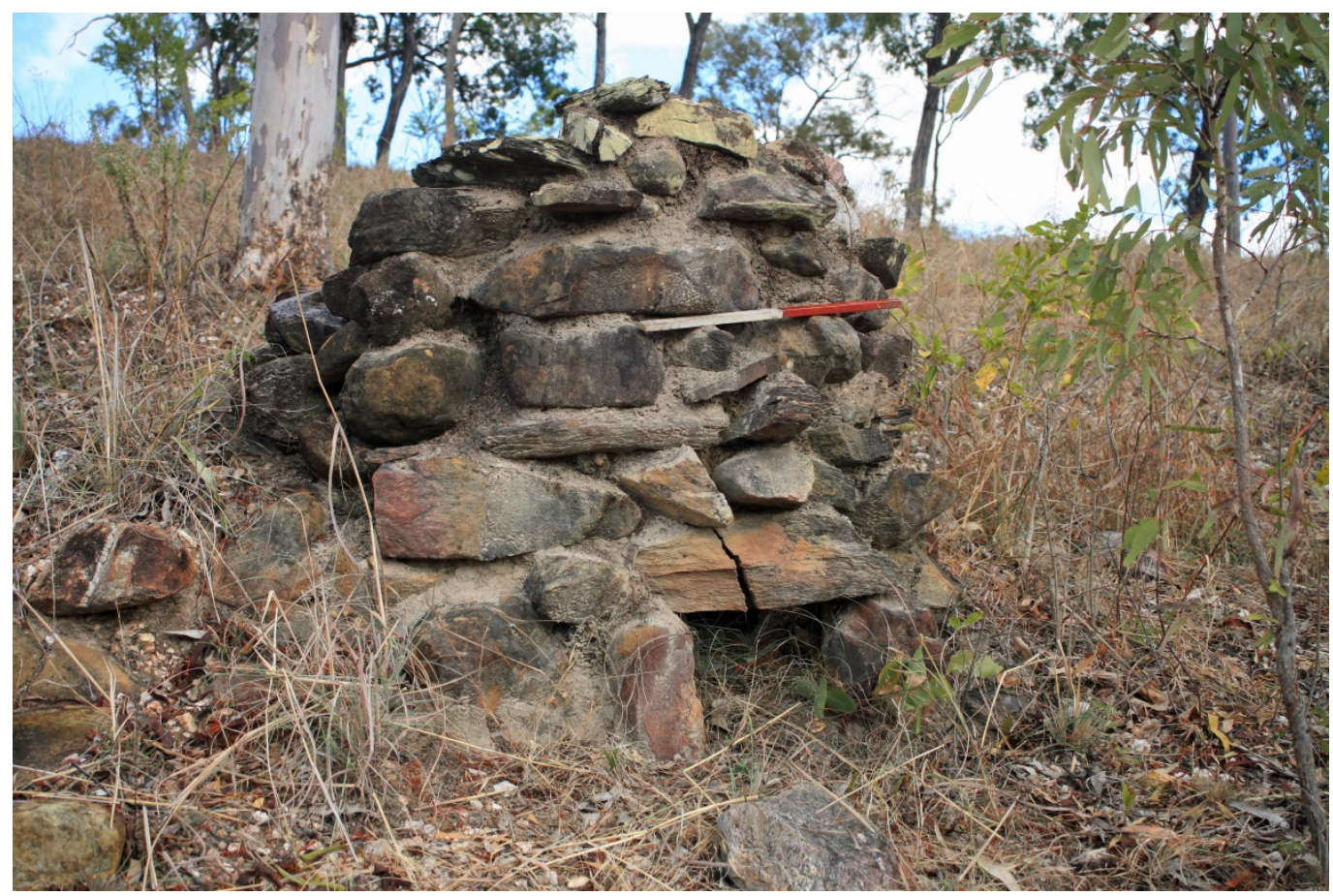

Figure 5. Although ovens are often associated with temple sites in FNQ, those on the Palmer are not associated with religious buildings (Photograph: Gordon Grimwade).

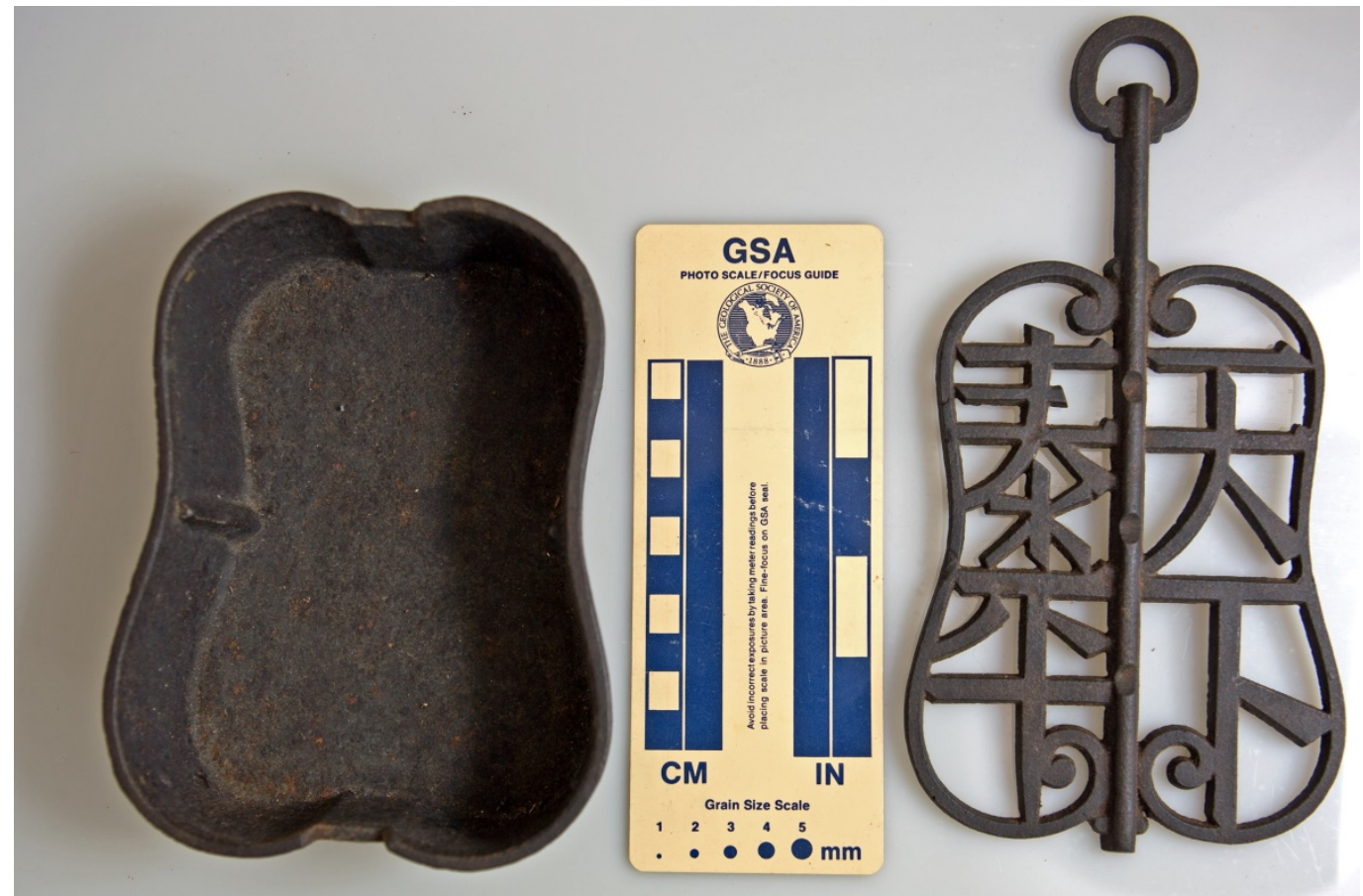

Figure 6. Metal baking dish obtained by a private collector c.1985 from the Palmer River Goldfield (Photograph: Gordon Grimwade).

The focus on the R16 historic reserve as the only mechanism by which archaeological assessments are triggered has a number of consequences for knowledge of the Chinese archaeology of the Palmer. Primarily compliance documents, impact assessments are necessarily limited in their descriptions, so, despite 22 years of work, the nature of, and variability in, site contents within R16 remains largely unknown. The area of R16 also represents only a small proportion of the total mining and other activity on the river and its tributaries (see Figure 3), leaving the precise nature and number of sites across the wider region also unknown. Of all work consulted for this study, Ah Toy's garden is the only project to be conducted outside the boundaries of R16. Finally, the limited application of the legislation to protect places of archaeological value and the even more limited resources to police them, has unfortunately made the Palmer a rich resource for hobbyist collectors. Often individual - and highly unique - artefacts, albeit with limited provenance, are passed on to archaeologists. One such example is a two-piece, cast, ferrous-metal container obtained from the Palmer by a bottle collector. The removable top is an imprint of four Chinese characters which translate as 'in peace all over the country'. It is believed to have been used as a bread-baking dish (Figure $6)$. 
Table 2. Archaeological reports for the Palmer River (R16).

\begin{tabular}{|c|c|c|c|c|c|c|c|c|c|c|c|c|}
\hline Date & Title & Area & Author & $\begin{array}{l}\text { Total } \\
\text { Sites }\end{array}$ & $\begin{array}{l}\text { Chinese } \\
\text { and } \\
\text { European }\end{array}$ & Chinese & Aboriginal & European & $\begin{array}{c}\text { Not } \\
\text { Definable }\end{array}$ & $\begin{array}{l}\text { Features/Artefacts } \\
\text { Recovered or Noted }\end{array}$ & Recommendations & $\begin{array}{l}\text { New } \\
\text { Sites } \\
\text { (Post- } \\
\text { 1991) } \\
\end{array}$ \\
\hline 1988 & $\begin{array}{l}\text { Historical Site } \\
\text { Survey for } \\
\text { Fortuna Pty } \\
\text { Ltd }\end{array}$ & $\begin{array}{l}\text { MLAs 1217, } \\
12911244\end{array}$ & $\begin{array}{l}\text { Grimwade } \\
\text { RCS Pty } \\
\text { Ltd }\end{array}$ & 18 & 0 & 2 & 0 & 0 & 16 & $\begin{array}{l}\text { 'Graves, stone structures, } \\
\text { Chinese ceramic bowl } \\
\text { (celadon)' }\end{array}$ & $\begin{array}{l}\text { Further recording of } \\
\text { majority of sites if they } \\
\text { were to be destroyed by } \\
\text { modern mining }\end{array}$ & - \\
\hline $\begin{array}{l}\text { Oct } \\
1988\end{array}$ & $\begin{array}{l}\text { Report on } \\
\text { Doughboy Ck } \\
\text { Gold Project }\end{array}$ & $\begin{array}{l}\text { MLAs } 1204 \\
12051206 \\
1207+ \\
\text { MCAs } 2800 \\
-21 \\
\end{array}$ & Alfredson & 21 & 0 & 0 & 2 & 0 & 19 & $\begin{array}{l}\text { 'Graves, glass, habitation } \\
\text { sites, scarred trees' }\end{array}$ & $\begin{array}{l}\text { Fence selected sites and } \\
\text { avoid. Retrieved artefacts } \\
\text { should be lodged with } \\
\text { Minister }\end{array}$ & - \\
\hline $\begin{array}{l}\text { Aug } \\
1989\end{array}$ & $\begin{array}{l}\text { EIS } \\
\text { Archaeology } \\
\text { Carpentaria } \\
\text { Gold }\end{array}$ & $\begin{array}{l}1255,1256 \\
12571258 \\
\text { (Cooktown) } \\
4189 \\
\text { (Mareeba) } \\
\end{array}$ & $\begin{array}{l}\text { Grimwade } \\
\text { RCS Pty } \\
\text { Ltd }\end{array}$ & 10 & 0 & 3 & 0 & 0 & 7 & $\begin{array}{l}\text { 'Opium tins, Schnapps } \\
\text { bottles, meat can, frypan, } \\
\text { celadon ceramic' }\end{array}$ & $\begin{array}{l}\text { Further recording } \\
\text { required if sites are to be } \\
\text { destroyed }\end{array}$ & - \\
\hline $\begin{array}{l}\text { Dec } \\
1990\end{array}$ & $\begin{array}{l}\text { EIS } \\
\text { Archaeology } \\
\text { EP 31/90 }\end{array}$ & $\begin{array}{l}\text { EP 31/90 H } \\
\text { Pawlaczyk }\end{array}$ & $\begin{array}{l}\text { Grimwade } \\
\text { RCS Pty } \\
\text { Ltd }\end{array}$ & 8 & 0 & 0 & 0 & 8 & 0 & $\begin{array}{l}\text { 'Shafts, stonewall, dam, } \\
\text { UK ‘Cable’ ceramic } \\
\text { pattern, fry pan, match } \\
\text { boxes' }\end{array}$ & $\begin{array}{l}\text { Exploratory work OK if it } \\
\text { avoids key areas. Further } \\
\text { mapping required before } \\
\text { mining }\end{array}$ & - \\
\hline 1990 & $\begin{array}{l}\text { Cambrian } \\
\text { Resources NL } \\
\text { Cradle Ck }\end{array}$ & & $\begin{array}{l}\text { Cambrian } \\
\text { Resources }\end{array}$ & 0 & 0 & 0 & 0 & 0 & 0 & & $\begin{array}{l}\text { EIS stated that an } \\
\text { archaeological study will } \\
\text { be completed. It inferred } \\
\text { habitation sites were } \\
\text { extant above flood levels } \\
\text { of creeks }\end{array}$ & - \\
\hline $\begin{array}{l}\text { Nov } \\
1990\end{array}$ & $\begin{array}{l}\text { EIS } \\
\text { Archaeology }\end{array}$ & $\begin{array}{l}13001319 \\
13231324 \\
13271350 \mathrm{~S} \\
\text { V Foster } \\
\end{array}$ & $\begin{array}{l}\text { Grimwade } \\
\text { RCS Pty } \\
\text { Ltd }\end{array}$ & 39 & 0 & 8 & 0 & 5 & 26 & $\begin{array}{l}\text { 'Mainly alluvial } \\
\text { workings and habitation } \\
\text { sites, opium bowl, } \\
\text { celadon' }\end{array}$ & $\begin{array}{l}\text { No impediment on } \\
\text { mining. Avoid if possible }\end{array}$ & - \\
\hline $\begin{array}{l}\text { Nov } \\
1990\end{array}$ & $\begin{array}{l}\text { EIS } \\
\text { Archaeology }\end{array}$ & $\begin{array}{l}\text { 6714 Chaos } \\
\text { S V Foster }\end{array}$ & $\begin{array}{l}\text { Grimwade } \\
\text { RCS Pty } \\
\text { Ltd }\end{array}$ & 3 & 0 & 1 & 0 & 1 & 1 & $\begin{array}{l}\text { 'Water race, habitation, } \\
\text { matchbox, shovel heads, } \\
\text { beer bottles' }\end{array}$ & $\begin{array}{l}\text { No impediment on } \\
\text { mining. Avoid if possible }\end{array}$ & - \\
\hline $\begin{array}{l}\text { Jul } \\
1991\end{array}$ & EIS & $\begin{array}{l}\text { ML } 3119 \text { (K } \\
\text { Lawton) }\end{array}$ & $\begin{array}{l}\text { Grimwade } \\
\text { RCS Pty } \\
\text { Ltd }\end{array}$ & 10 & 0 & 1 & 0 & 6 & 3 & $\begin{array}{l}\text { 'Domestic sites, road } \\
\text { works, forge, hotel' }\end{array}$ & $\begin{array}{l}\text { Avoid if possible. Retain } \\
7 \text { sites }\end{array}$ & 0 \\
\hline $\begin{array}{l}\text { Mar } \\
1993\end{array}$ & $\begin{array}{l}\text { EIS Cultural } \\
\text { Resources }\end{array}$ & ML 40056 & $\begin{array}{l}\text { Grimwade } \\
\text { RCS Pty } \\
\text { Ltd }\end{array}$ & 8 & 0 & 4 & 0 & 3 & 1 & $\begin{array}{l}\text { 'Rifle range, domestic } \\
\text { sites, dam, stone } \\
\text { pitching' }\end{array}$ & $\begin{array}{l}\text { Avoid if possible. Retain } \\
1 \text { site (rifle range) }\end{array}$ & 5 \\
\hline
\end{tabular}




\begin{tabular}{|c|c|c|c|c|c|c|c|c|c|c|c|c|}
\hline Date & Title & Area & Author & $\begin{array}{l}\text { Total } \\
\text { Sites }\end{array}$ & $\begin{array}{l}\text { Chinese } \\
\text { and } \\
\text { European }\end{array}$ & Chinese & Aboriginal & European & $\begin{array}{c}\text { Not } \\
\text { Definable }\end{array}$ & $\begin{array}{l}\text { Features/Artefacts } \\
\text { Recovered or Noted }\end{array}$ & Recommendations & $\begin{array}{l}\text { New } \\
\text { Sites } \\
\text { (Post- } \\
\text { 1991) } \\
\end{array}$ \\
\hline $\begin{array}{l}\text { Apr } \\
1993\end{array}$ & $\begin{array}{l}\text { EIS Cultural } \\
\text { Resources }\end{array}$ & $\begin{array}{l}\text { ML } 40050 \\
\text { and access } \\
\text { update } \\
\text { ML3054 }\end{array}$ & $\begin{array}{l}\text { Grimwade } \\
\text { RCS Pty } \\
\text { Ltd }\end{array}$ & 11 & 0 & 7 & 0 & 0 & 4 & $\begin{array}{l}\text { ‘Graves, domestic sites, } \\
\text { dam’ }\end{array}$ & $\begin{array}{l}\text { 'Retain graves, alluvial } \\
\text { workings can be } \\
\text { destroyed' }\end{array}$ & 2 \\
\hline $\begin{array}{l}\text { Apr } \\
1993\end{array}$ & $\begin{array}{l}\text { EIS Cultural } \\
\text { Resources }\end{array}$ & $\begin{array}{l}\text { ML 3075 - } \\
77,6719 \text {, } \\
\text { 7355, 40057 } \\
\text { (Thomson, } \\
\text { Thomson \& } \\
\text { Jarman) }\end{array}$ & $\begin{array}{l}\text { Grimwade } \\
\text { RCS Pty } \\
\text { Ltd }\end{array}$ & 18 & 2 & 7 & 0 & 6 & 3 & $\begin{array}{l}\text { 'Dwelling sites, hard } \\
\text { rock mine, battery, } \\
\text { graves' }\end{array}$ & $\begin{array}{l}\text { 'Retain Cat } 1 \text { sites } \\
\text { (compulsory), avoid } \\
\text { others as possible' }\end{array}$ & 10 \\
\hline $\begin{array}{l}\text { Jul } \\
1993\end{array}$ & $\begin{array}{l}\text { Cultural } \\
\text { Heritage Study }\end{array}$ & $\begin{array}{l}\text { ML } 40034 \& \\
\text { access } \\
\text { (McGrath) }\end{array}$ & $\begin{array}{l}\text { Grimwade } \\
\text { RCS Pty } \\
\text { Ltd }\end{array}$ & 9 & 0 & 5 & 0 & 1 & 3 & $\begin{array}{l}\text { 'Graves, stone structures, } \\
\text { stone pitching' }\end{array}$ & $\begin{array}{l}\text { 'Retain graves, avoid } \\
\text { stone pitching and taped } \\
\text { sites' }\end{array}$ & 5 \\
\hline $\begin{array}{l}\text { Aug } \\
1993\end{array}$ & $\begin{array}{l}\text { Cultural } \\
\text { Heritage Study }\end{array}$ & $\begin{array}{l}\text { ML } 6787 \\
678840023- \\
25(\mathrm{~S} \mathrm{~V} \\
\text { Foster) } \\
\end{array}$ & $\begin{array}{l}\text { Grimwade } \\
\text { RCS Pty } \\
\text { Ltd }\end{array}$ & 19 & 0 & 4 & 0 & 1 & 14 & $\begin{array}{l}\text { 'Domestic sites, alluvial } \\
\text { workings, campsite, } \\
\text { graves' }\end{array}$ & $\begin{array}{l}\text { 'Retain graves, avoid } \\
\text { stone pitching and taped } \\
\text { sites' }\end{array}$ & 16 \\
\hline $\begin{array}{l}\text { Sep } \\
1994\end{array}$ & $\begin{array}{l}\text { Cultural } \\
\text { Heritage Study }\end{array}$ & $\begin{array}{l}\text { ML } 40053 \& \\
\text { access }\end{array}$ & $\begin{array}{l}\text { Grimwade } \\
\text { RCS Pty } \\
\text { Ltd }\end{array}$ & 2 & 0 & 0 & 0 & 1 & 1 & $\begin{array}{l}\text { 'Reef mine, stone } \\
\text { pitched bench' }\end{array}$ & Avoid & 2 \\
\hline $\begin{array}{l}\text { Oct } \\
1994\end{array}$ & $\begin{array}{l}\text { A Survey of } \\
\text { MLAs } 2938 \\
\text { and } 2939 \\
\text { Palmer } \\
\text { Goldfield } \\
\end{array}$ & $\begin{array}{l}\text { MLAs } 2938 \\
\text { and } 2939 \\
\text { (Donam } \\
\text { P/L) }\end{array}$ & Meiklejohn & 8 & 3 & 2 & 0 & 2 & 1 & $\begin{array}{l}\text { 'Graves, township, } \\
\text { battery, road' }\end{array}$ & $\begin{array}{l}\text { Avoid Binnies Battery. } \\
\text { No recommendations re } \\
\text { other sites }\end{array}$ & 0 \\
\hline $\begin{array}{l}\text { Nov } \\
1995\end{array}$ & $\begin{array}{l}\text { A Survey of } \\
\text { ML } 40022 \\
\text { Palmer } \\
\text { Goldfield } \\
\end{array}$ & $\begin{array}{l}\text { ML } 40022 \\
\text { (Stamnas, } \\
\text { Walker, } \\
\text { Zoppani) }\end{array}$ & Meiklejohn & 4 & 0 & 0 & 0 & 0 & 4 & Alluvial workings & Avoid forge & 0 \\
\hline $\begin{array}{l}\text { Sep } \\
1996\end{array}$ & $\begin{array}{l}\text { CH } \\
\text { Assessment } \\
\text { MLs } 20266 \\
\text { and } 20267 \\
\end{array}$ & $\begin{array}{l}\text { ML } 20266 \\
\text { and } 20267 \\
\text { (C } \\
\text { Fitzgerald) }\end{array}$ & $\begin{array}{l}\text { Grimwade } \\
\text { GG \& A }\end{array}$ & 6 & 0 & 1 & 0 & 0 & 5 & $\begin{array}{l}\text { 'Stone pitching, } \\
\text { habitation site, grave' }\end{array}$ & $\begin{array}{l}\text { Retain stone pitching and } \\
\text { grave }\end{array}$ & 6 \\
\hline $\begin{array}{l}\text { Feb } \\
2001\end{array}$ & $\begin{array}{l}\text { CH } \\
\text { Assessment } \\
\text { MLs 20309 } \\
\text { and } 20311 \\
\end{array}$ & $\begin{array}{l}\text { ML } 20309 \\
\text { and } 20311 \\
\text { (DeLacy) }\end{array}$ & $\begin{array}{l}\text { Grimwade } \\
\text { \& Burke } \\
\text { GG\&A }\end{array}$ & 2 & 0 & 1 & 0 & 1 & 0 & 'Dam, habitation site' & Avoid & 0 \\
\hline $\begin{array}{l}\text { Mar } \\
2001\end{array}$ & $\begin{array}{l}\text { CH } \\
\text { Assessment } \\
\text { MLs } 20272 \\
\text { and } 20283 \\
\end{array}$ & $\begin{array}{l}\text { ML } 20309 \\
\text { and 20311 } \\
\text { (DeLacy) }\end{array}$ & $\begin{array}{l}\text { Grimwade } \\
\text { \& Burke } \\
\text { GG\&A }\end{array}$ & 8 & 0 & 1 & 0 & 5 & 2 & $\begin{array}{l}\text { 'Stone pitching, } \\
\text { alluvials, dam, habitation } \\
\text { site' }\end{array}$ & $\begin{array}{l}\text { Variously destroy or } \\
\text { avoid }\end{array}$ & 0 \\
\hline
\end{tabular}




\begin{tabular}{|c|c|c|c|c|c|c|c|c|c|c|c|c|}
\hline Date & Title & Area & Author & $\begin{array}{l}\text { Total } \\
\text { Sites }\end{array}$ & $\begin{array}{l}\text { Chinese } \\
\text { and } \\
\text { European }\end{array}$ & Chinese & Aboriginal & European & $\begin{array}{c}\text { Not } \\
\text { Definable }\end{array}$ & $\begin{array}{l}\text { Features/Artefacts } \\
\text { Recovered or Noted }\end{array}$ & Recommendations & $\begin{array}{l}\text { New } \\
\text { Sites } \\
\text { (Post- } \\
\text { 1991) }\end{array}$ \\
\hline $\begin{array}{l}\text { Aug } \\
2007\end{array}$ & $\begin{array}{l}\mathrm{CH} \\
\text { Assessment } \\
\text { Palmer } \\
\text { Goldfield ML } \\
20433\end{array}$ & $\begin{array}{l}\text { ML } 20433 \\
\text { (T J Bidner) }\end{array}$ & $\begin{array}{l}\text { Grimwade } \\
\text { GG\&A }\end{array}$ & 2 & 0 & 0 & 0 & 0 & 2 & 'Water race, forge' & Avoid & 0 \\
\hline $\begin{array}{l}\text { Aug } \\
2007\end{array}$ & $\begin{array}{l}\text { CH } \\
\text { Assessment } \\
\text { Palmer } \\
\text { Goldfield ML } \\
2032020330\end{array}$ & $\begin{array}{l}\text { ML } 20320 \\
20330 \mathrm{~F} \\
\text { Zopponi }\end{array}$ & $\begin{array}{l}\text { Grimwade } \\
\text { GG\&A }\end{array}$ & 9 & 1 & 4 & 0 & 3 & 1 & $\begin{array}{l}\text { 'Dam, walk track, } \\
\text { habitation, stone } \\
\text { pitching' }\end{array}$ & Avoid & 0 \\
\hline $\begin{array}{l}\text { Aug } \\
2007\end{array}$ & $\begin{array}{l}\text { CH } \\
\text { Assessment } \\
\text { Palmer } \\
\text { Goldfield ML } \\
\text { 20408, 20420, } \\
\text { 20421, } 20422 \\
20453\end{array}$ & $\begin{array}{l}\text { ML 20408, } \\
20420, \\
20421, \\
2042220453 \\
\text { (R } \\
\text { Fitzgerald) }\end{array}$ & $\begin{array}{l}\text { Grimwade } \\
\text { GG\&A }\end{array}$ & 7 & 0 & 1 & 0 & 5 & 1 & $\begin{array}{l}\text { 'Stone pitching, costean, } \\
\text { alluvial workings, shaft' }\end{array}$ & Avoid & 0 \\
\hline $\begin{array}{l}\text { Aug } \\
2007\end{array}$ & $\begin{array}{l}\text { CH } \\
\text { Assessment } \\
\text { Palmer } \\
\text { Goldfield } \\
\text { ML20396, } \\
\text { 20409, 20410, } \\
\text { 20430, 20431, } \\
\text { 20432, 20480 }\end{array}$ & $\begin{array}{l}\text { ML 20396, } \\
20409, \\
20410, \\
20430, \\
20431, \\
20432, \\
20480 \text { (M } \\
\text { Vaughan) }\end{array}$ & $\begin{array}{l}\text { Grimwade } \\
\text { GG\&A }\end{array}$ & 8 & 0 & 3 & 0 & 3 & 2 & $\begin{array}{l}\text { 'Stone pitching, } \\
\text { habitation site, alluvial } \\
\text { workings, reef mine' }\end{array}$ & Avoid where necessary & 0 \\
\hline $\begin{array}{l}\text { Oct } \\
2010\end{array}$ & $\begin{array}{l}\text { CH } \\
\text { Assessment } \\
\text { Palmer } \\
\text { Goldfield } \\
\text { ML20411, } \\
20412,20588, \\
20589\end{array}$ & $\begin{array}{l}\text { ML 20411, } \\
20412, \\
20588, \\
20589 \text { (C \& } \\
\text { R } \\
\text { Fitzgerald) }\end{array}$ & $\begin{array}{l}\text { Grimwade } \\
\text { GG\&A }\end{array}$ & 5 & 0 & 1 & 0 & 0 & 4 & & & 0 \\
\hline & Total & & & 235 & 6 & 56 & 2 & 51 & 120 & & & 46 \\
\hline
\end{tabular}




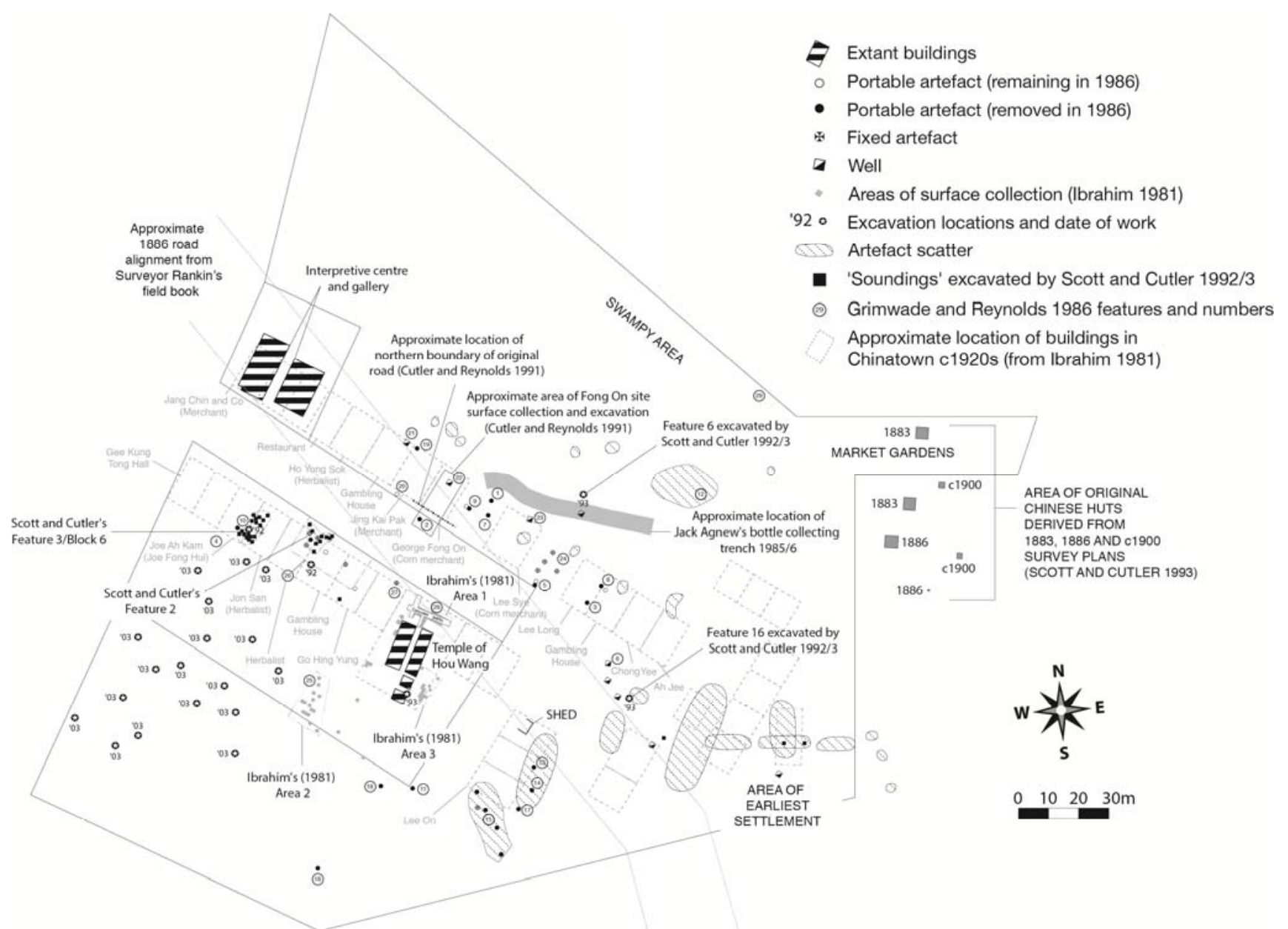

Figure 7. Archaeological work conducted at Atherton Chinatown 1981-2003.

Cooktown (1874-1930s)

While earlier work (Grimwade and Meiklejohn 1993) noted the existence of the Chinese shrine at the cemetery and the 'former town dump' as areas of Chinese activity, the first detailed work on Cooktown took place via Rains' (1999, 2005) and McCrae's (2001) studies on the town dump. Rains completed two theses on this area, which together constitute the most comprehensive study of the Chinese social and economic landscape of Cooktown. He recorded 46 sites, including irrigation and drainage systems associated with market gardening (mainly wells, ponds or small dams), hut sites and surface concentrations (Rains 2005:221-228). Rains' project did not include excavation and in comparison to the detailed focus on identifiable Chinese commodities, other artefact types (such as European alcohol bottle glass) were only cursorily noted. Amongst the ceramics recorded by McCrae and Rains were fragments of a large, globular bulk storage jar with similarities to the Marteban jars used by Chinese and Macassan traders in Southeast Asia to ship bulk foodstuffs. This form has not been noted in New Zealand, the United States or southern Australia and appears to be restricted to sites in northern and northwest Australia (McCrae 2001:19-20), leading Rains (2005:285) to speculate that it was a form of multifunctional bulk container specific to Southeast Asian trade networks.

Between 2003 and 2005 monitoring and salvage archaeology was undertaken in connection with a sewerage scheme involving over $50 \mathrm{~km}$ of underground pipes through areas of high archaeological potential (Rowney and Grimwade 2004). One of these was Burke \& Grimwade
Adelaide Street, where the Chinese community had congregated in the late nineteenth century (Grimwade et al. 2007). Although artefacts were only retrieved from the trenches, rather than excavated archaeologically, over 700 diagnostic Chinese and European artefacts were catalogued and deposited with the James Cook Museum, Cooktown (Grimwade 2007:66). Two years later, however, a fire destroyed the collection.

\section{Atherton Chinatown (1877-1920s)}

There have been at least nine archaeological projects at Atherton Chinatown. The first focussed on selected areas where 'obvious artefacts were located' (Ibrahim 1981:101), including around the Temple, and near a well. All artefacts were collected from $10 \mathrm{~m}$ x $10 \mathrm{~m}$ squares, but little analysis was conducted on the results. Ibrahim noted that the artefacts from the rear of the Temple included a high percentage of European alcohol bottles, and were possibly the result of festival celebrations at the Temple complex (Ibrahim 1981:114). Five years later, Grimwade and Reynold's 1986 project was the first comprehensive survey of the majority of the former township. They located and plotted 29 individual features, from wells and remnant vegetation to fixed and portable artefacts (Figure 7). During this work 105 artefacts were removed to the care of the Material Culture Unit, James Cook University. In the same year an energetic bottle collector was given access to a significant proportion of the northern part of the site by the then owner, seriously compromising the archaeology. 


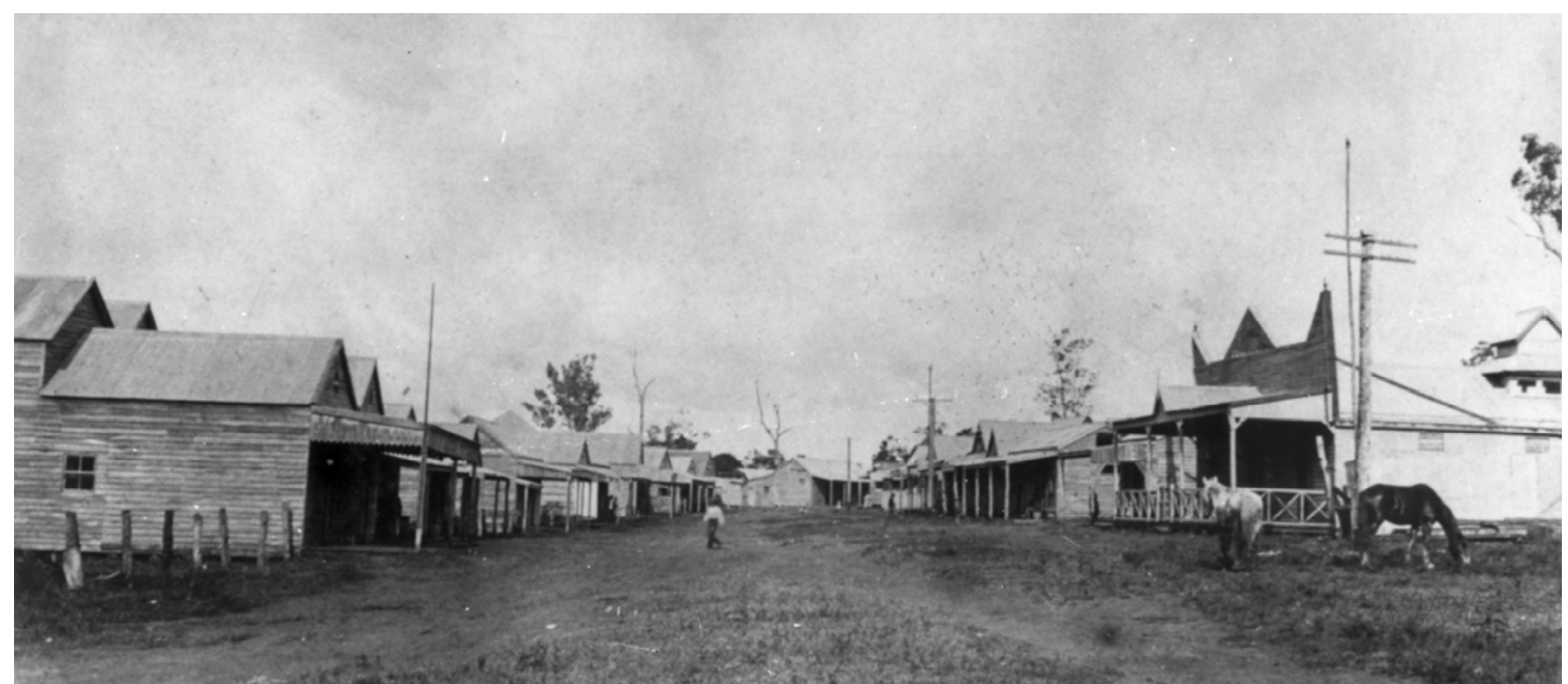

Figure 8. Atherton Chinatown's main street c.1912. The street primarily contained retail stores with attached residences. Excavations in 2003 investigated the rear yards of the buildings to the right (Photograph: Courtesy National Trust of Queensland).

Subsequent excavations in 1991-1993 by Scott, Cutler and Reynolds (Cutler and Reynolds 1991; Scott and Cutler 1992, 1993) targeted two areas with high potential for subsurface deposits: the site of the Fong On store, and the eastern end of the town where the first settlement had been located. This eastern end contained the greatest density of material (Scott and Cutler 1993). The most notable feature was what may have been a deliberately dug storage pit or back-filled well (Feature 16 on Figure 7 ), the assemblage from which included over 500 fragments of ceramic, Chinese coins, cattle, pig and horse bone fragments, and what was possibly the remains of one or more large metal boxes (Scott and Cutler 1993:66).

Three things are notable about work at Atherton Chinatown. Firstly, much early work was carried out piecemeal, using idiosyncratic excavation techniques and backed by a research agenda that is no longer apparent. Scott, Cutler and Reynold's various excavations, for example, were extremely limited in scope (mostly targeting foundation stumps) and area (less than $1 \%$ of the Fong On store site, for example) that little information was forthcoming, although the disturbed nature of the site and the dependency on limited funding would have significantly contributed to data limitations. Secondly, no studies attempted to answer spatial or behavioural research questions, other than in extremely general terms (e.g. where were buildings located? or what goods did people discard on this site?). Further work in the late 1990s and early 2000s was designed to address specific structural questions or general conservation and management issues rather than archaeological research questions, and deliberately avoided unnecessary subsurface disturbance, an approach that has been maintained by the National Trust to the present. Thirdly, no detailed artefact analyses were conducted at the time, although this situation has been partially rectified by an Honours project that catalogued and briefly analysed 602 glass and ceramic artefacts drawn from all previous studies (Dunk 2010).
In part to address previous deficiencies, the most recent archaeological work in 2003 was oriented towards determining the nature and extent of the archaeological resources in the southern portion of the site and the degree to which occupation could be demonstrated away from the main street (Rowney 2003). Eighteen $1 \mathrm{~m} \times 1 \mathrm{~m}$ squares were excavated across the southwestern corner of the town in what would have been rear yards for structures fronting the southern side of the main street (Figure 8). The assemblage of $1500+$ artefacts was dominated by alcohol bottles (beer and wine) and Chinese ceramics, with only small proportions of European tea and tableware.

\section{Cairns Chinatown (1882-1930s)}

While studied extensively by historian Cathy May (1984, 2001) and more recently by Sandi Robb (2012), there have been only two excavations on this site. The first testpitted several adjoining allotments between Grafton and Sheridan Streets in 2001 (Burke and Grimwade 2001) and the second excavated 17 pile pits and service trenches across the same area. The Grafton Street half of this block was part of Chinatown from at least 1885 until 1936. While only 68 artefacts were recovered from the initial test pits, their distribution indicated that the oldest came from the centre of the block, roughly associated with the rear yards of former structures. Although representing only $2.3 \%$ of the total site, subsequent excavations (Grimwade and Rowney 2004) recovered a total of 3469 artefacts. Two different assemblages were apparent from the 2004 excavations, correlating roughly with the Grafton Street (former Chinatown) (assemblage 1) and Sheridan Street (European) (assemblage 2) sides respectively. The ceramics in assemblage 1 were mostly typical Chinese tablewares and domestic food storage ('brownware') vessels (62\% of all ceramics), with few, if any English or European earthenwares or porcelain tablewares. 


\section{Croydon Chinatown (1880-c.1936)}

Work at the Croydon Chinatown site was undertaken in 2000 to identify the approximate boundary of the former settlement and fence it for use as a tourist attraction, as well as to protect it for future, more detailed, archaeological research. Archaeological work was limited to surface recording of artefacts and collection of key artefacts prior to the installation of a walking trail. Unfortunately, very little information can be extracted from these data, since the trail was deliberately sited through the areas with the lowest density of, and fewest diagnostic, artefacts. Only 38 items were collected from this work, although more were recorded.

Artefacts from this site included ceramic teapot fragments with the 'simple flower' design (Ritchie 1986:226), a pattern which appears to be unusual on FNQ Chinese sites (Grimwade 2003:56). Seven ‘funs’ or opium measurement trays made from small, deliberate offcuts from brass opium canisters and domestic food cans were also noted. While their size (averaging $4 \mathrm{~cm}$ long $\mathrm{x} 3.4 \mathrm{~cm}$ wide) is consistent with examples recovered elsewhere (e.g. Ritchie 1986:387), they do not exhibit the characteristic diagonal or edge folds typical of this artefact type. These are probably funs tray 'blanks'; that is, objects that have been partially modified in preparation for later use (Figure 9) (P. Wegars, pers. comm., 2013).

\section{Comparing Chinese Sites in FNQ}

While there are an estimated 50 consultancy reports and theses deriving from work in the region between 1980 and 2013, the sources consulted for this project were both inconsistent and largely incomparable. For data derived from ceramics, for example, only two variables have been recorded consistently across all reports: the colour of applied decoration and fragment location (rim, base, body). Diagnostic details are often absent, even from excavated collections, including assessments of paste colour, composition, wall thickness, or conjoins and size calculations from rim sherds - all useful details for reconstructing a minimum vessel count (MVC). Identifications of vessel sizes and specific decorative patterns are also commonly generalised: rice bowls, for example, are sometimes assigned to a subjective size category - 'large' or 'small' - but mostly referred to generically, without quantifying rim diameter and rarely referring to size standards, such as Ritchie (1986). Terminology for ceramic forms also varies, and definitions are not usually explicit. Conjoin analysis is not a common procedure, resulting in an inability to identify larger, more readily classifiable, portions of broken vessels, although this would not necessarily be possible in all cases. In addition, while the three main hand-painted decorative types (Bamboo, Double Happiness and Four Seasons) are usually recognised, patterns are often described only according to their colour or motifs, precluding identification of other types and making it impossible to be certain that all examples of the three main types have been positively identified.

This has repercussions for site comparisons. Firstly, accurate minimum vessel counts are impossible for most sites; the only projects explicitly to construct MVCs that could be considered representative are Dunk (2010) and Rains (2005). Dunk's MVC was calculated for both broad vessel forms (e.g. plates vs bowls) and basic decorative

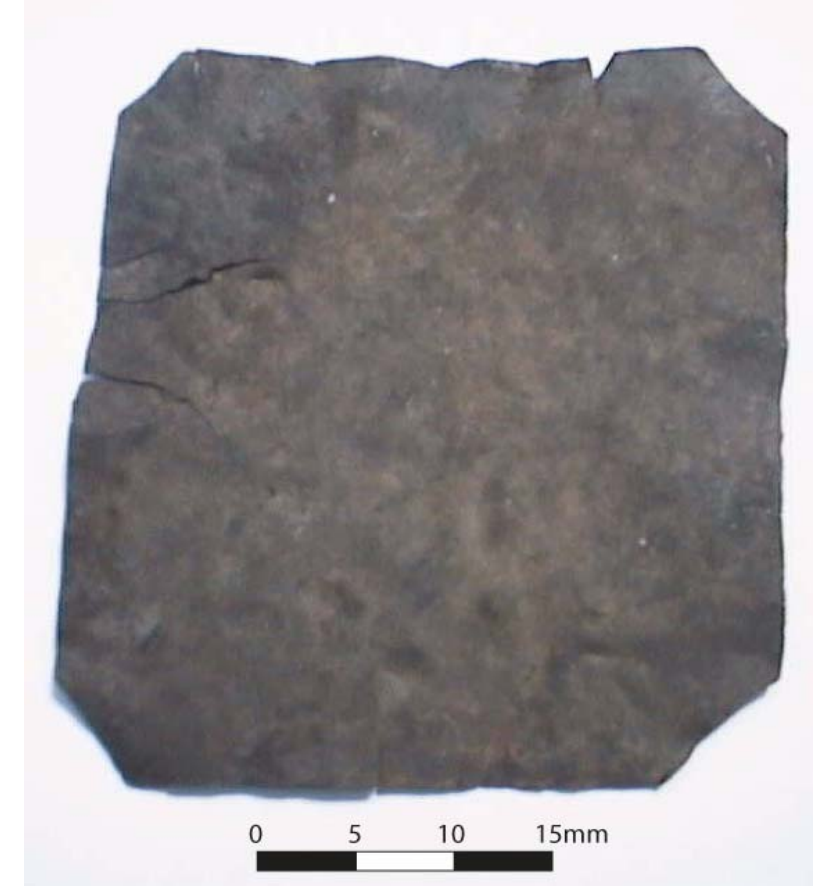

Figure 9. One of the 'funs' tray blanks from Croydon Chinatown (Photograph: Heather Burke).

categories, however she provides very little specific data on individual pieces, precluding identification of different vessel sizes amongst her data. Secondly, lack of conjoining as a practice has no doubt meant that bases identified in catalogues as 'celadon', and which may well have belonged to hand painted vessels originally, may have been misattributed as plain celadon here.

As a result, MVC counts for this paper have been calculated conservatively: for bottles the diagnostic element was bases, unless a higher MVC could be obtained from another element - for example, seals for gin bottles and spouts for spouted jars (soy sauce pots) but all other ceramic MVCs were calculated on bases, unless definite descriptions of rim size or decorative pattern variations allowed for more discerning counts. Pattern types were only identified from definite catalogue entries or photographs.

\section{Glass and Ceramics: The Behaviours of Drinking and Dining \\ Alcohol Consumption in FNQ}

Table 3 illustrates comparisons in alcohol-related bottle glass and ceramics between FNQ sites. Across these sites it is clear that European alcohols were far more common than traditional Chinese forms, and beer was more common than spirits, although this is less noticeable when data for Chinese and European spirits are combined. Both Atherton Chinatown and Ah Toy's Garden show the addition of imported Asian beer and soft drink (Mitusya cider) brands from the late nineteenth century onwards (Ross 2009:10). The issue for analysis becomes whether or not a dominance of European alcohols reflects a degree of accommodation, greater availability, the maintenance of traditional practices, or something else. 
Table 3. Minimum vessel counts for alcohol containers and contents, Overseas Chinese sites, FNQ.

\begin{tabular}{|c|c|c|c|c|c|c|c|}
\hline Site & Context & Date & Chinese Alcohol & European Alcohol & $\begin{array}{l}\text { Japanese and } \\
\text { Other Alcohol }\end{array}$ & Reference & Comments \\
\hline Atherton Chinatown & Town & 1877-1920s & Liquor bottles (1) & $\begin{array}{l}\text { Wine/champagne (3) } \\
\text { Beer (3) } \\
\text { Schnapps (1) } \\
\text { Gin (1) }\end{array}$ & $\begin{array}{l}\text { Dai Nippon beer (2) } \\
\text { San Miguel } \\
\text { (Philippine) beer (2) } \\
\text { Kirin beer (1) }\end{array}$ & Dunk 2010 & $\begin{array}{l}\text { Temple still in use until } \\
\text { 1960s }\end{array}$ \\
\hline Cairns Chinatown & Town & 1882-1930s & Liquor bottles (13) & $\begin{array}{l}\text { Wine/champagne (77) } \\
\text { Beer (18) } \\
\text { Gin (19) } \\
\text { Whiskey/distilled } \\
\text { liquor (16) } \\
\text { Wine (12) } \\
\text { Ink (4) } \\
\text { Schnapps (1) }\end{array}$ & None & $\begin{array}{l}\text { Grimwade and } \\
\text { Rowney } 2004\end{array}$ & $\begin{array}{l}\text { Includes American, Danish } \\
\text { and German beer, English } \\
\text { and Dutch gin. } \\
\text { [44] = equal apportionment } \\
\text { of bottles identified as soft } \\
\text { drink/beer in catalogue. } \\
\text { Condiments mostly vinegar. } \\
\text { Includes } 12 \text { cut-off (and } \\
\text { reused?) bottle bases. }\end{array}$ \\
\hline Cooktown & $\begin{array}{l}\text { Market } \\
\text { gardening }\end{array}$ & 1873-1935 & $\begin{array}{l}\text { Liquor bottles (10) } \\
\text { Globular jars (4) }\end{array}$ & $\begin{array}{l}\text { No quantities given, } \\
\text { but includes Dutch gin, } \\
\text { schnapps, brandy, beer } \\
\text { and wine }\end{array}$ & - & Rains 2005 & $\begin{array}{l}\text { Data from mixed rubbish } \\
\text { dumps (surface scatter } \\
\text { only). } \\
\text { Globular jars thought to } \\
\text { contain alcohol }\end{array}$ \\
\hline $\begin{array}{l}\text { Ah Toy’s Garden, } \\
\text { Palmer River }\end{array}$ & $\begin{array}{l}\text { Mining } \\
\text { and } \\
\text { market } \\
\text { gardening }\end{array}$ & 1883-1934 & - & $\begin{array}{l}\text { No quantities given, } \\
\text { but included Dutch } \\
\text { schnapps, Scotch } \\
\text { whisky, English gin, } \\
\text { Dutch gin and German } \\
\text { beer }\end{array}$ & $\begin{array}{l}\text { Sakura beer } \\
\text { San Miguel } \\
\text { (Philippine) beer }\end{array}$ & Jack et al. 1984 & $\begin{array}{l}\text { No MVC data available. } \\
\text { Includes } 1 \text { cut-off (and } \\
\text { reused?) bottle base. }\end{array}$ \\
\hline
\end{tabular}




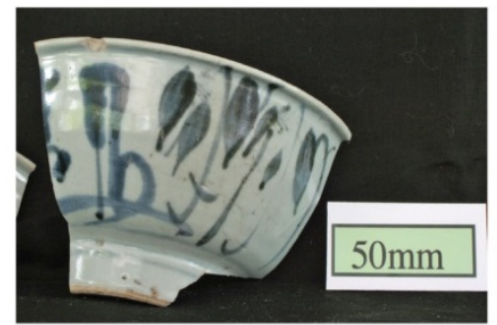

a. Bamboo pattern rice bowl, Cooktown

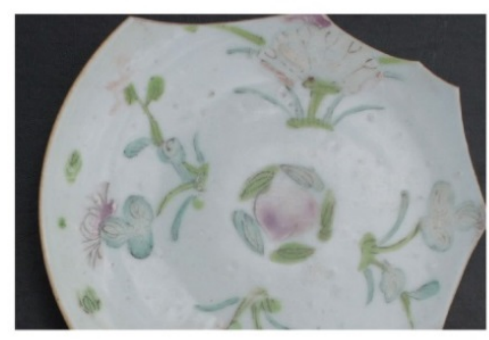

c. Four Seasons pattern plate, Cooktown

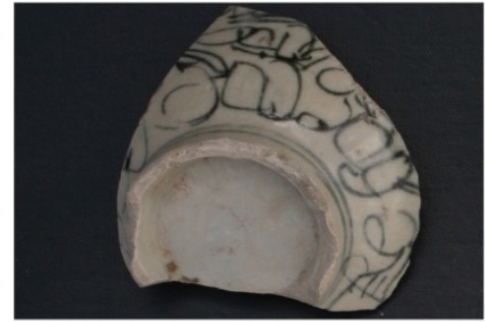

b. Double Happiness pattern rice bowl, Cooktown

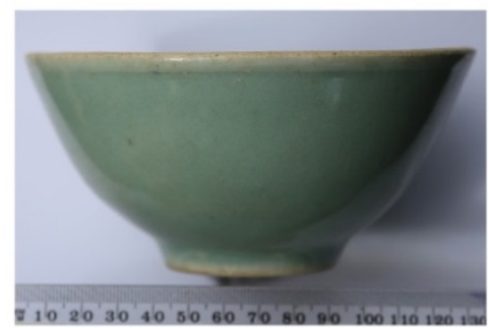

d. Plain celadon rice bowl,

Atherton Chinatown

Figure 10. The four main decorative ceramic types (Photographs: Gordon Grimwade).

In some ways this is a typical problem. When Ritchie conducted his studies of nineteenth century sites in New Zealand he noted that European alcohols tasted quite different from Chinese varieties, and argued that their presence was therefore evidence of acculturation in drinking habits (Ritchie 1986:657-658). Ross (2010), in noting similar preferences for early twentieth century sites in Canada, however, argued that, by then, ordinary Chinese were already familiar with European alcohols in China and therefore this exposure helped direct their choices. European wines, beers and liquors had certainly been introduced to China in the mid-nineteenth century via the treaty ports but it was not until 1903 that beer became the first Western-style alcohol to be brewed and distributed in China (Ross 2010:237). European alcohols had thus become popular amongst the wealthy elite by the 1880s (Ross 2010:237), but were not widely consumed by ordinary people until the twentieth century.

Ross' (2010) argument fits into a much later picture of movements and choices compared to earlier Australian and New Zealand sites. Rains' analysis of the import trends for Cooktown indicates quite clearly that a preference for importing European alcohols existed both before and after the main influx of people and the establishment of a monthly steamer service from China in 1875 (Rains 2005:240). In 1874 Chinese import lists favoured European case alcohol, the most common of which was brandy, followed by stout, ale, whisky and gin, porter and champagne (Rains 2005:242). This was similar to European import profiles and after 1875 the range of choices simply expanded to encompass (in order of quantity) European brandy, Chinese wine, European schnapps, wine, stout, ale, claret, gin, rum and port (Rains 2005:248). Given that the highly efficient, reliable and regular merchant supply networks that buttressed Overseas Chinese communities in Australia (Bowen 2011:27) would have made access to either European or traditional alcohols equally straightforward, local merchants could have imported traditional liquors just as easily, suggesting that the consumption of European alcohols was a deliberate preference rather than an accommodative mechanism based on geographicallylimited or European-controlled trading networks. Data from FNQ sites suggest that large quantities of European alcohols were being widely and deliberately consumed by ordinary Chinese as early as the 1870s; a practice which is unlikely to represent accommodation or acculturation under these circumstances.

While we cannot know what differing alcohols 'meant' to the Overseas Chinese, in one respect drinking was probably more a male and working class activity than an 'ethnic' one per se (Ross 2010:248). In other words, it was linked to the work and recreation patterns of labourers and the construction of masculinity more generally in colonial contexts (e.g. Moore 1998). It is tempting to speculate that better data might reveal patterns between the range and quantities of alcohols consumed in different contexts, for example hierarchically, in urban versus isolated sites, or according to different levels of cross-cultural interaction between Chinese and non-Chinese populations, but at present this remains unknown.

\section{Ceramic Use in FNQ}

The range of Chinese ceramics at FNQ sites (Table 4) is dominated by large, medium and small bowls (particularly rice bowls and including serving bowls), followed by tea and/or wine cups and spoons. Other forms, such as small dishes, cooking pots or plates, are rare. This range is typical of expatriate Chinese assemblages elsewhere, indicating foodways based around traditional, communal soups and stews served in large bowls, but consumed from smaller individual bowls along with rice and condiments. In terms of decorative types, FNQ sites contain higher proportions of plain celadon and Four Seasons wares in contrast to Double Happiness or Bamboo designs (Figure 10), although the failure of previous studies to classify decorative patterns more exhaustively probably means that the MVCs for plain celadon - arguably the most easily recognisable decorative type - may have been inflated here. 


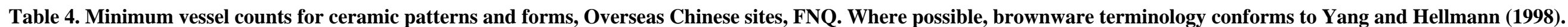

\begin{tabular}{|c|c|c|c|c|c|c|c|}
\hline Site & Context & Date & $\begin{array}{c}\text { Chinese Tableware } \\
\text { Patterns }\end{array}$ & $\begin{array}{c}\text { Chinese Tableware } \\
\text { Forms }\end{array}$ & $\begin{array}{c}\text { Brownware and } \\
\text { Other Forms }\end{array}$ & Reference & Comments \\
\hline Atherton Chinatown & Town & 1877-1920s & $\begin{array}{l}\text { Plain celadon (19) } \\
\text { Four Seasons (12) } \\
\text { Double Happiness (1) }\end{array}$ & $\begin{array}{l}\text { Bowl (13) } \\
\text { Rice bowl (8) } \\
\text { Teacup (7) } \\
\text { Spoon (5) } \\
\text { Teapot (4) } \\
\text { Wine cup (2) }\end{array}$ & $\begin{array}{l}\text { Spouted jar (9) } \\
\text { Liquor bottle (1) }\end{array}$ & $\begin{array}{l}\text { Dunk 2010; } \\
\text { Rowney 2003 }\end{array}$ & $\begin{array}{l}\text { MVCs from diagnostic artefacts } \\
\text { catalogued by Dunk (sample of } 602 \text { of } \\
\text { total collection only). }\end{array}$ \\
\hline $\begin{array}{l}\text { Cairns Chinatown } \\
\text { (Grafton Street side) }\end{array}$ & Town & 1882-1930s & $\begin{array}{l}\text { Plain celadon (29) } \\
\text { Four Seasons (7) } \\
\text { 'Blue on white' (4) } \\
\text { Double Happiness (1) } \\
\text { Bamboo (1) }\end{array}$ & $\begin{array}{l}\text { Standard rice bowl (11) } \\
\text { Tea cup (6) } \\
\text { Small bowl (6) } \\
\text { Bowl (unspecified) (4) } \\
\text { Large serving bowl (4) } \\
\text { Spoon (4) } \\
\text { Medium serving bowl (2) } \\
\text { Large plate (2) } \\
\text { Wine cup (2) } \\
\text { Large rice bowl (1) } \\
\text { Small plate (1) } \\
\text { Small dish (1) }\end{array}$ & $\begin{array}{l}\text { Spouted jar (35) } \\
\text { Food storage (7) } \\
\text { Large storage vessel (5) } \\
\text { Water storage (4) } \\
\text { Teapot (3) } \\
\text { Cooking pot (2) }\end{array}$ & $\begin{array}{l}\text { Grimwade } \\
\text { and } \\
\text { Rowney } 2004\end{array}$ & $\begin{array}{l}\text { MVCs recalculated from catalogue. } \\
\text { Majority of patterns described but not } \\
\text { identified (e.g. ‘blue on white'). } \\
\text { Size classes for bowls recalculated from } \\
\text { catalogue according to Ritchie (1986:207, } \\
\text { 217). }\end{array}$ \\
\hline Cooktown (general) & Market gardens & $1873-1935$ & $\begin{array}{l}\text { Plain celadon (30) } \\
\text { Bamboo (6) } \\
\text { Simple Flower (3) } \\
\text { Four Seasons (2) }\end{array}$ & $\begin{array}{l}\text { Spoon (12) } \\
\text { Medium bowl (10) } \\
\text { Rice bowl (9) } \\
\text { Cup (3) } \\
\text { Large bowl (2) } \\
\text { Vase (1) }\end{array}$ & $\begin{array}{l}\text { Ovoid jar (15) } \\
\text { Liquor bottle (10) } \\
\text { Barrel jar (7) } \\
\text { Food storage (6) } \\
\text { Spouted jar (5) } \\
\text { Globular jar (4) } \\
\text { Cooking pot (3) }\end{array}$ & Rains 2005 & $\begin{array}{l}\text { Not all rubbish associated with Chinese } \\
\text { residents? } \\
\text { Surface material only. }\end{array}$ \\
\hline $\begin{array}{l}\text { Cooktown (Adelaide } \\
\text { Street Chinatown) }\end{array}$ & Town & 1873-1930s & $\begin{array}{l}\text { Plain celadon (11) } \\
\text { Bamboo (13) } \\
\text { Four Seasons (9) } \\
\text { Decorated celadon (6) } \\
\text { Double Happiness (1) }\end{array}$ & $\begin{array}{l}\text { Rice bowl (25) } \\
\text { Shallow dish (7) } \\
\text { Spoon (3) } \\
\text { Large bowl (2) } \\
\text { Wine cup (1) }\end{array}$ & $\begin{array}{l}\text { Spouted jar (4) } \\
\text { Barrel jar (3) } \\
\text { Food storage (3) } \\
\text { Liquor bottle (3) } \\
\text { Storage jar lid (2) } \\
\text { Bulk pourer container } \\
\text { (soy sauce?) (1) } \\
\text { Teapot (1) } \\
\text { Teacup (1) } \\
\text { Ovoid jar (1) } \\
\text { Round jar (1)* } \\
\end{array}$ & $\begin{array}{l}\text { Grimwade } \\
\text { et al. } 2007\end{array}$ & $\begin{array}{l}\text { Numerous European artefacts in this } \\
\text { collection that do not necessarily represent } \\
\text { Chinese residency. }\end{array}$ \\
\hline $\begin{array}{l}\text { Ah Toy's Garden, } \\
\text { Palmer River }\end{array}$ & $\begin{array}{l}\text { Mining and } \\
\text { market gardening }\end{array}$ & $1883-1934$ & Patterns not identified & Rice bowl & - & $\begin{array}{l}\text { Jack et al. } \\
1984\end{array}$ & $\begin{array}{l}\text { No quantities given, but they note a } \\
\text { general dearth of ceramics. }\end{array}$ \\
\hline $\begin{array}{l}\text { Palmer River } \\
\text { (general) }\end{array}$ & $\begin{array}{l}\text { Mining, towns and } \\
\text { market gardening }\end{array}$ & 1873-1930s & $\begin{array}{l}\text { Plain celadon } \\
\text { Four Seasons } \\
\text { Bamboo } \\
\text { Double Happiness (1 } \\
\text { fragment) } \\
\end{array}$ & Not specified & Not specified & Comber 1991 & $\begin{array}{l}\text { Patterns noted in order of frequency (plain } \\
\text { celadon most frequent, Double Happiness } \\
\text { least), but no MVCs or fragment counts. } \\
\text { No details of form provided in the report. }\end{array}$ \\
\hline
\end{tabular}


A continued adherence to Chinese cultural preferences for food is in contrast to the patterns observed in alcohol consumption. This suggests that concepts of identity may have been constructed differently in each arena, although this may well have varied hierarchically (e.g. between merchants and labourers. For an account of how merchants deliberately incorporated European ceramics and dining patterns, see Lydon 1999), and was perhaps linked to the densely layered medicinal and health connotations traditionally ascribed to food (Rains 2005:340).

It is also worth noting that the decorative forms observed in FNQ were probably among the more expensive decorative types available. While there are no comparative figures for Australia, an analysis of inventory records for Californian sites has indicated that plain celadon and Four Seasons wares cost more than either the Bamboo or Double Happiness designs (Sando and Felton 1993:165). Sando and Felton (1993) used this trend to predict that greater quantities of bowls and cheaper patterns will be found on more mobile sites (such as mining camps) and a wider range of forms and more expensive patterns on more stable sites (such as Chinatowns). The three key sites of Atherton, Cairns and Cooktown were all urban centres and seem to conform to this expectation (i.e. they contain more plain celadon and Four Seasons wares), however Comber (1991:27) also noted these types as the two most common on the more isolated sites of the Palmer, although without providing any details. A more targeted collection of detailed ceramic data across urban and rural sites would cast more light on this question, and perhaps highlight variations that are unobservable in the current data. Rains (2005:299) makes the important point that, like European working class communities, Overseas Chinese communities in Australia generally tended to be more affluent in comparison to their counterparts in rural China, suggesting that nuances of class or status can only be more subtly signalled with better comparative data.

Unlike studies in Canada and the US (e.g. Hellmann and Yang 1997; Michaels 2005; Ross 2010; van Bueren 2008), no individually peck-marked ceramics are currently known from FNQ (or indeed, Australian) sites, removing one possible means to speculate on notions of individual versus group identity. Michaels (2005), for example, found pecked markings on both Chinese and European wares from the Market Street Chinatown site in San Francisco (1866-1887). Vessels from boarding house contexts contained pecked names and nicknames which were interpreted to indicate personalisation and individual ownership, while vessels recovered near stores tended to be marked with characters signifying group blessings at communal meals. Van Bueren (2008:84) found similar pecked blessings on the interior of a white earthenware European plate from a Californian farm feature dating to the 1850s-1880s, the use of which was interpreted as a communal serving vessel. In contrast, Ross (2010:247) at Lion Island, British Columbia, noted personal ownership marks on two porcelain liquor cups but none of the rice bowls from the same site, implying that, in this context, social drinking was a more personalised activity than dining.

\section{Conclusion}

Historical archaeology indicates that the Overseas Chinese in FNQ appear to have behaved distinctively in several ways: they were ready adopters of European alcohols, while continuing to maintain cultural preferences regarding food, their temples were often adaptations of traditional forms, and their practice of building pig ovens in the nineteenth century part of a distinctive trans-Tasman pattern. This suggests that their constructions of personal and communal identity were being fashioned in different ways, although the limited interpretation that can be derived from the current data highlights critical gaps that need to be filled before historical archaeological research can contribute further to understanding why, where and how such distinctions arose.

Several questions arise because of the tentative nature of the present assessment. Why did Chinese immigrants retain their traditional dining habits while quickly absorbing European alcohols? How did Chinese preferences in dining or drinking (or other activities) change over time or between different groups? Why were 'celadon wares' apparently more favoured than patterned tablewares? Were they cheaper and more readily available or were they related in any way to the origins of the users? Given the extreme mobility evident in ordinary life (amongst Overseas Chinese as well as Europeans), are expectations about more or less mobility and different assemblage compositions borne out? To what degree are patterns of alcohol consumption related to gender or class in the colonial context rather than ethnicity? How were changing patterns of individual or communal identity signalled in Australia? At present, none of these questions can be answered.

Four things are evident from this. Firstly, consultancy work needs to take comparative questions surrounding consumption (between Europeans and Overseas Chinese, between status groups within Overseas Chinese communities, or between early and later Chinese communities) to future projects, so that new data can begin to contribute to a more structured understanding of variation, regional trends and significance. Secondly, given that the data presented in this paper would undoubtedly be different if the original assemblages were reanalysed consistently and more exhaustively, a concerted programme to revisit previous artefact collections according to the same methods and more detailed sets of variables is clearly warranted. Thirdly, the plethora of 'grey literature' material - particularly for the Palmer River - requires consolidation and development to provide a research and management tool that can generate more useful outcomes. Lastly, case-specific (and ideally contrasting) studies are needed that can question the construction of race, class and social identity as concepts that currently guide study of the Chinese diaspora, as well as interrogating how these concepts changed across time, space and social context. Such an archaeology needs to compare Chinese sites, not just with European ones, but also with each other across time and space to investigate the heterogeneity that needs to be brought out within groups (Mullins 2008:154). This can only be achieved through targeted and controlled excavations at selected sites, combined with detailed artefact studies and contextual biographies of people, place and kin. Only 
through such studies will we advance knowledge beyond basic presence/absence questions and begin to try and understand the complex lives of people whose material traces have generated so much description, but generally eluded detailed interpretation.

\section{Acknowledgements}

We would like to thank Martin Rowney, who worked on both the Atherton and Cairns Chinatown projects, Melissa Dunk, who provided her Excel spreadsheet of the collated Atherton Chinatown data, along with a copy of her thesis, Kevin Rains and Sandi Robb, both of whom provided copies of their respective theses, and Patricia Wegars, who identified the potential funs tray blanks. Sean Ulm suggested the original idea for this paper and the editors and reviewers offered insightful comments that have materially contributed to the final product.

\section{References}

Bell, P. 1995 Chinese ovens on mining settlement sites in Australia. In P. Macgregor (ed.), Histories of the Chinese in Australia and the South Pacific: Proceedings of an International Public Conference held at the Museum of Chinese Australian History, Melbourne, 8-10 October 1993, pp.213-229. Melbourne: Museum of Chinese Australian History.

Bell, P. 1997 History of the Chinese in Australia. Unpublished report to Australian Heritage Commission.

Bjornskov, M. 2001 Rock, mortar and traditions: An archaeological study of Chinese 'ovens' in the Northern Territory. In C. Fredericksen and I. Walters (eds), Altered States: Material Culture Transformations in the Arafura Region, pp.121-147. Darwin: Northern Territory University Press, Darwin.

Bowen, A. 2011 The merchants: Chinese social organisation in colonial Australia. Australian Historical Studies 42:25-44.

Burke, H. and G. Grimwade 2001 Rusty's Markets ReDevelopment Site Archaeological Report. Unpublished report to Gleeson Consulting Pty Ltd.

Chan, H. 1999 The identity of the Chinese in Australian history. Queensland Review 6(2):1-10.

Chan, H. 2007 Qiaoxiang and the diversity of Chinese settlement in Australia and New Zealand. In T. Chee-Beng (ed.), Chinese Transnational Networks, pp.153-171. London: Routledge.

Comber, J. 1991 Palmer Goldfield Heritage Sites Study (Stage 2). Unpublished report to Queensland Department of Environment and Heritage.

Comber, J. 1995 The Palmer Goldfield. Australasian Historical Archaeology 13:41-48.

Curthoys, A. 2001 "Men of all nations, except Chinamen": Europeans and Chinese on the goldfields of New South Wales. In I. McCalman, A. Cook and A. Reeves (eds), Gold: Forgotten Histories and Lost Objects of Australia, pp.103123. Cambridge: Cambridge University Press.

Cutler, B. and B. Reynolds 1991 Report on Atherton Chinatown, North Queensland: The Fong-On Family Residence Preliminary Excavations. Unpublished report to Material Culture Unit, James Cook University.

Dunk, M. 2010 Made in China: An Analysis of the Artefact Assemblage from Atherton Chinatown, North Queensland. Unpublished BA (Hons) thesis, Department of Archaeology, La Trobe University, Melbourne.
Fitzgerald, J. 2005 Revolution and respectability: Chinese Masons in Australian history. In A. Curthoys and M. Lake (eds), Connected Worlds: History in Transnational Perspective, pp.89-110. Canberra: ANU E Press.

Fitzgerald, J. 2007 Big White Lie: Chinese Australians in White Australia. Sydney: University of New South Wales Press.

Gojak, D. 2009 Old Marulan 2007 Archaeological Investigations - Final Report. Vol. 2. Unpublished report to Umwelt Pty Ltd and Cemex Pty Ltd.

Government Statistician 2013 Population by census district, Queensland 1861-1911, Historical Tables, Demography, 1823-2008 (Q150 Release). Retrieved 20 September 2013 from http://www.oesr.qld.gov.au/products/tables/historicaltables-demography/index.php.

Grimwade, G. 1990 Palmer Goldfields Cultural Resources. 3 vols. Unpublished report to Queensland Department of Environment and Heritage.

Grimwade, G. 2003 Gold, gardens, temples and feasts: Chinese temple, Croydon, Queensland. Australasian Historical Archaeology 21:50-57.

Grimwade, G. 2007 Cooktown sewerage scheme: The world's longest archaeological trench? In K. Wong Hoy (ed.), Rediscovered Past: China in North Queensland, pp.65-74. North Melbourne: Chinese Heritage in North Queensland Inc (CHINA Inc).

Grimwade, G. 2008 Crispy roast pork: Using Chinese Australasian pig ovens. Australasian Historical Archaeology 26:21-28.

Grimwade, G. and A. Meiklejohn 1993 Cooktown Heritage Study. Vol. 2. Unpublished report to Cook Shire Council.

Grimwade, G. and B. Reynolds 1986 Report on Atherton Chinatown, North Queensland: Site Survey. Unpublished report to Material Culture Unit, James Cook University of North Queensland.

Grimwade, G. and M. Rowney 2004 Rusty’s Markets ReDevelopment Site Archaeological Excavation Report. Unpublished report to Gilligan's Development Pty Ltd.

Grimwade G., M. Rowney and I. McCrae 2007 Salvage Archaeology Cooktown Sewerage Scheme. Unpublished report to Cook Shire Council.

Hellmann, V.R. and J.K. Yang 1997 Previously undocumented Chinese artifacts. In M. Praetzellis and A. Praetzellis (eds), Historical Archaeology of an Overseas Chinese Community in Sacramento, California: The Archaeological Excavations, pp.155-202. Vol. 1. Rohnert Park, CA: Anthropological Studies Center, Sonoma State University Academic Foundation Inc.

Historical Census and Colonial Data Archive 2013 Queensland Census Data. Retrieved 20 September 2013 from http://hccda.ada.edu.au/regions/QLD

Hum Lee, R. 1960 The Chinese in the United States of America. Hong Kong: Hong Kong University Press.

Ibrahim, A.L.H. 1981 Hou Wang Miau: A Study of the Material Culture of the Chinese Temple, Atherton. Unpublished Grad. Dip. thesis, James Cook University, Cairns.

Jack, R.I., K. Holmes and R. Kerr 1984 Ah Toy's garden: A Chinese market garden on the Palmer River goldfield. Australian Journal of Historical Archaeology 2:51-58.

Jack, R.L. 1896 Map of the Palmer District Compiled by R.L. Jack, Government Geologist from Surveys and Sketches by W.T. White, J.T. Embley, J.J. Davies, P.F. Selheim, E.A. 
Kayser, H. Rosser and R.L. Jack. Brisbane: Geological Survey Office.

Jack, R.L. 1899 Report on a Visit to the Palmer Goldfield. Brisbane: Government Printer.

Jack, R.L. 1921 Northmost Australia: Three Centuries of Exploration, Discovery and Adventure in and Around the Cape York Peninsula Queensland. London: Simpkin, Marshall, Hamilton, Kent \& Co.

Jones, T.G. 1997 The Chinese in the Northern Territory. Darwin: Northern Territory University Press.

Kirkman, N. 1984 The Palmer River Goldfield 1873-1883. Unpublished BA (Hons) thesis, History Department, James Cook University.

Kirkman, N. 1993 From minority to majority: Chinese on the Palmer River gold-field, 1873-1876. In H. Reynolds (ed.), Race Relations in North Queensland, pp.243-257. Townsville: Department of History and Politics, James Cook University.

Lydon, J. 1999 Many Inventions: The Chinese in the Rocks 1890-1930. Melbourne: Monash Publications in History.

Mainery, M. 2001 Fuel for the Fire: Chinese Cooking Features in California. Paper presented to Society for Historical Archaeology Annual Meeting, Long Beach, California.

May, C. 1984 Topsawyers: The Chinese in Cairns 1870-1920. Cairns: Department of History and Politics, James Cook University.

May, C. 1993a The Chinese in Cairns district 1876-1920. In H. Reynolds (ed.), Race Relations in North Queensland, pp.258-275. Cairns: Department of History and Politics, James Cook University.

May, C. 2001 Chinese in Queensland. In J. Jupp (ed.), The Australian People: An Encyclopaedia of the Nation, its People and their Origins, pp.210-213. Cambridge: Cambridge University Press.

McRae, I. 2001 Canton to Cooktown: A Study of Chinese Ceramic Shipping Containers. Unpublished Grad. Dip. in Archaeological Heritage thesis, Department of Archaeology and Palaeoanthropology, University of New England, Armidale.

Michaels, G. 2005 Peck-marked vessels from the San Jose Market Street Chinatown: A study of distribution and significance. International Journal of Historical Archaeology 9(2):123-134.

Moore, C. 1998 Colonial manhood and masculinities. Journal of Australian Studies 22(56):35-50.

Mullins, P. 2008 “The strange and unusual”: Material and social dimensions of Chinese identity. Historical Archaeology 42(3):152-157.

Pan, L. (ed.) 1998 The Encyclopaedia of the Chinese Overseas. Surrey: Curzon Press.

Queensland Registrar General's Office 1881 Census of Queensland 1881. Brisbane: Government Printer. Retrieved 20 September 2013 from http://hccda.ada.edu.au/documents/

Rains, K. 1999 Rice Bowls and Beer Bottles: Processual and Postprocessual Approaches to Explaining the Material Culture of Late Nineteenth and Early Twentieth Century Overseas Chinese Ethnicity in Far North Queensland. Unpublished BA (Hons) thesis, School of Social Science, The University of Queensland, Brisbane.
Rains, K. 2005 Intersections: The Overseas Chinese Social Landscape of Cooktown, 1873-1935. Unpublished PhD thesis, School of Social Science, The University of Queensland, Brisbane.

Reeves, K. 2010 Sojourners or a new diaspora? Economic implications of the movement of Chinese miners to the south-west Pacific goldfields. Australian Economic History Review 50(2):178-192.

Reeves, K. and B. Mountford 2011 Sojourning and settling: Locating Chinese Australian history. Australian Historical Studies 42(1):111-125.

Ritchie, N. 1986 Archaeology and History of the Chinese in Southern New Zealand during the Nineteenth Century: A Study of Acculturation, Adaptation and Change. Unpublished PhD thesis, Department of Anthropology and Archaeology, University of Otago, Dunedin.

Robb, S. 2012 Cairns Chinatown: A Heritage Study: A History of the Cairns Chinese Community. Cairns: Cairns and District Chinese Association Inc.

Ross, D.E. 2009 Identification and dating of Japanese glass beverage bottles. Technical Briefs in Historical Archaeology 4:7-17.

Ross, D.E. 2010 Comparing the material lives of Asian transmigrants through the lens of alcohol consumption. Journal of Social Archaeology 10(2):230-254.

Rowney, M. 2003 Atherton Chinatown Archaeological Excavation Report. Unpublished report to National Trust of Queensland.

Rowney M. and G. Grimwade 2004 Cooktown Sewerage Scheme Background Research. Unpublished report to Cook Shire Council.

Sando, R.A. and D.L. Felton 1993 Inventory records of ceramics and opium from a nineteenth-century Chinese store in California. In P. Wegars (ed.), Hidden Heritage: Historical Archaeology of the Overseas Chinese, pp.151-176. Amityville: Baywood Publishing Company.

Scott, B. and B. Cutler 1992 Atherton Chinatown - Interim Report. Unpublished report to Material Culture Unit, James Cook University.

Scott, B. and B. Cutler 1993 Atherton Chinatown, North Queensland: Archaeological Site Survey. Unpublished report to Material Culture Unit, James Cook University.

van Bueren, T.M. 2008 Late nineteenth-century Chinese farm workers in the California Mother Lode. Historical Archaeology 42(3):80-96.

Voss, B. and R. Allen 2008 Overseas Chinese archaeology: Historical foundations, current reflections, and new directions. Historical Archaeology 42(3):5-28.

Williams, M. 1999 Brief sojourn in your native land: Sydney links with south China. Queensland Review 6(2):11-23.

Wong Hoy, K. 2004 Thursday Island en route to citizenship and the Queensland goldfields: Chinese aliens and naturalised British subjects, 1879-1903. Journal of Australian Colonial History 6:159-174.

Yang, J.K. and V. R. Hellmann 1998 What's in the pot? An emic study of Chinese brown glazed stoneware. Proceedings of the Society for California Archaeology 11:59-66. 
